\title{
Une pause dans la marche vers la civilisation des loisirs ?
}

\author{
Alain Chenu et Nicolas Herpin*
}

L'avènement d'une « civilisation des loisirs » favorisée par la croissance économique, annoncée au début des années 1960 par le sociologue Joffre Dumazedier, est démentie par les faits, si l'on se réfère aux constats dressés à partir des enquêtes Emploi du temps de 1974, 1986 et 1998.

La tendance séculaire à la baisse de la durée du travail s'est interrompue entre les deux dernières enquêtes. En contrepartie, la durée du temps de loisir marque le pas dans le cas des actifs occupés, et ne continue à augmenter que dans celui des inactifs. Les femmes étaient par le passé très défavorisées par rapport aux hommes pour ce qui est de la durée des loisirs. Ce handicap a été en partie rattrapé par l'atténuation des disparités des temps de travail professionnel et domestique avec les hommes. Le temps libre féminin progresse, mais au détriment du travail domestique, et non de l'activité professionnelle. Alors qu'en 1974, les milieux populaires bénéficiaient de moins de loisirs que les milieux favorisés, c'est aujourd'hui l'inverse, mais cette progression résulte pour l'essentiel de leur plus grande exposition au chômage, et de l'extension de celui-ci.

Le diplôme est le déterminant de la durée du travail dont l'impact a le plus changé : ce sont maintenant les plus diplômés qui travaillent le plus, et qui consacrent le moins de temps aux loisirs. La différenciation des pratiques de loisirs selon le niveau scolaire conserve les mêmes caractéristiques principales en 1974 et en 1998 : les spectacles et sorties, la participation à la vie associative, la lecture, la pratique des jeux ou de la musique sont plutôt l'apanage des plus diplômés. Les téléspectateurs sont encore plus souvent que par le passé les titulaires du certificat d'études et les sans diplôme : l'avance en la matière de ces derniers, par rapport aux bacheliers et aux diplômés de l'enseignement supérieur, s'est accrue.

* Alain Chenu fait partie du CREST-Insee (Laboratoire de sociologie quantitative), Nicolas Herpin du département des prix à la consommation, des ressources et des conditions de vie des ménages de l'Insee, et du CNRS.

Les noms et dates entre parenthèses renvoient à la bibliographie en fin d'article. 
$\mathbf{L}$ a « révolution culturelle du temps libre » (Dumazedier, 1988), fait partie des objectifs communément attribués à la société industrielle ou post-industrielle. Cependant, les enquêtes Emploi du temps, réalisées en France en 1974, en 1986 et en 1998 (avant que n'entrent en application les mesures de « Réduction du Temps de Travail »), font apparaître des évolutions qui ne confirment qu'imparfaitement la tendance à la croissance de la durée des loisirs et, de façon concomitante, la baisse de celle du travail aux âges actifs.

\section{La durée des loisirs des actifs en emploi a cessé d'augmenter au cours des dix dernières années}

Observés sur la population urbaine des 18-64 ans (cf. encadré 1), les loisirs augmentent entre 1974 et 1998 de près d'une heure par jour : ils passent de $23 \mathrm{~h} 06$ par semaine à $29 \mathrm{~h} 42$ (cf. tableau 1). L'évolution n'a cependant pas la même ampleur au cours des deux périodes de douze ans. L'augmentation de la durée des loisirs est deux fois plus forte entre 1974 et 1986 qu'entre 1986 et 1998. Cet accroissement est en grande partie lié à l'évolution du temps de travail professionnel. Celui-ci diminue d'un peu plus d'une demi heure par jour, passant de $32 \mathrm{~h} 24$ par semaine à $29 \mathrm{~h}$ dans le dernier quart du siècle. Le ralentissement dans la période récente doit aussi être mis en rapport avec l'évolution différente de la durée du travail professionnel au cours des deux sous-périodes. Après avoir diminué pendant la première période, cette durée augmente légèrement dans la seconde (de 28 h 42 en 1986 à 29 h en 1998). Ce renversement de la tendance séculaire à la baisse n'est pas propre à la France. Il concerne également d'autres pays industrialisés - notamment le Canada (Schor, 1990 ; Robinson et Godbey, 1999 ; Gershuny, 2000 : p. 177).

L'évolution des emplois du temps combine trois types d'effets. La participation à l'emploi d'abord. Dans le dernier quart du $\mathrm{XX}^{\mathrm{e}}$ siècle, elle diminue parmi les 18-64 ans (cf. tableau 2). Certes le taux d'emploi des femmes augmente continûment au cours de la période examinée. Mais la division par deux de la proportion des femmes au foyer - de $17 \%$ à $8 \%$ - ne suffit pas à compenser l'ampleur prise par les autres catégories d'inoccupés. La proportion des 18-64 ans au chômage passe de $2 \%$ en 1974 à $10 \%$ en 1998, celle des étudiants de ces tranches d'âge, de $4,5 \%$ à $10 \%$. Cette période se caractérise enfin par le quasi-doublement de la proportion des retraités et de pré-retraités - de $4 \%$ à $7 \%$. En conséquence, la part de la population (Chenu, 2001) en emploi à l'âge actif diminue de $70 \%$ en 1974 à $61,5 \%$ en 1998.

Le développement du temps partiel au détriment du temps plein contribue aussi à faire reculer la part du travail dans les emplois du temps. Le temps partiel ne concernait que 5,2\% des actifs employés en 1974 ; cette proportion triple en 1998 et atteint $16,6 \%$.

Le troisième élément est la durée du travail professionnel des actifs en emploi. Chaque statut d'emploi, bien qu'obéissant à des réglementations collectives, n'interdit pas à l'employeur de rechercher une certaine flexibilité en offrant au travailleur les moyens de moduler sa durée de travail : heures supplémentaires pour le temps plein, travail rapporté au domicile notamment pour les enseignants, définition individualisée du temps partiel, seconde activité professionnelle, allongement non rémunéré du temps passé au travail, au-delà des durées conventionnelles, notamment (mais pas exclusivement) pour les cadres, liberté encore plus grande pour le travail des indépendants. Cette modulation « contractuelle» de la durée du travail ne se réduit pas aux différences du statut de l'emploi. Elle s'y ajoute pour en émousser les contours. Or cette composante évolue aussi à la baisse, du moins pendant la première période examinée (cf. tableau 1). La semaine de travail (1) des actifs à plein temps (la catégorie la plus nombreuse) atteint 44 h 24 en 1974 ; elle baisse de trois heures, jusqu'à 41 h 24, en 1986, mais elle augmente de plus d'une heure en 1998 (42 h 36). Leurs loisirs, dont la durée hebdomadaire progresse entre 1974 et 1986 de plus de trois heures et demie, n'augmentent plus dans la période suivante. Chez les 18-64 ans employés à temps partiel, la durée du travail augmente sur les deux périodes. Elle passe de 22 h 12 en 1974 à 24 h 42 en 1986 et à 29 h 12 en 1998 : les horaires des personnes à temps partiel, de plus en plus nombreuses, se rapprochent de ceux des actifs occupés à temps plein.

Chez les chômeurs, les étudiants, les retraités, les femmes au foyer, la durée du temps de loisir s'est accrue davantage que chez les personnes en emploi (cf. tableau 1). Le temps libre hebdomadaire des personnes hors emploi progresse de cinq heures de 1974 à 1986 , et de cinq nouvelles

1. Il s'agit de la semaine de travail au sens large, y compris les déplacements domicile-travail et la formation professionnelle. 
Encadré 1

\section{LA MÉTHODE DU CARNET D'ACTIVITÉS ET LES ENQUÊTES EMPLOI DU TEMPS}

Une enquête Emploi du temps (EDT) est centrée sur un carnet d'activités, c'est-à-dire sur un questionnaire permettant de recueillir des récits de journées dans un format présentant trois caractéristiques principales :

- la durée observée est généralement de vingt-quatre heures ;

- le questionnement est ouvert : les répondants définissent leurs activités dans les termes de leur choix ;

- pour chaque ligne d'activité, des questions annexes sont posées : question ouverte sur l'activité secondaire éventuelle, questions fermées sur le lieu, sur les personnes en présence desquelles elle est exercée, et éventuellement sur le but de l'activité.

Les enquêtes réalisées par l'Insee font appel à la méthode dite "du lendemain ». Lors d'une première visite, l'enquêteur délivre les consignes de remplissage du carnet qu'il récupère deux ou trois jours plus tard (la « méthode de la veille », moins coûteuse parce que ne nécessitant qu'une interview, en face à face ou au téléphone, sollicite davantage la mémoire des répondants). Les questionnaires sur papier, autoadministrés, impliquent la spécification d'un pas, c'est-à-dire d'une unité de temps minimale correspondant à une ligne du formulaire - en 1974 et en 1986, le pas est de cinq minutes, en 1998, de dix (1) (cf. tableau A).

Pour chacune de ces trois enquêtes, la collecte a été étalée sur une durée d'un an, ce qui permet de carac- tériser les variations saisonnières des emplois du temps. Elle est toutefois interrompue pendant deux semaines fin décembre, et deux semaines début août. Par ailleurs, les répondants ne sont interrogés que s'ils sont présents dans leur résidence principale au moment de l'enquête. Les ménages collectifs - prisons, hôpitaux, etc. - sont hors champ. Toutes ces caractéristiques contribuent à une sous-évaluation de la part du loisir dans l'ensemble des activités.

Les non-réponses sont souvent le fait de personnes très mobiles pour des raisons professionnelles, de personnes vivant seules et donc plus difficiles à joindre, de personnes qui refusent l'enquête parce qu'elles sont peu ou pas alphabétisées, de personnes vivant dans des zones où l'insécurité est grande. Les jeunes adultes isolés, les individus de très faible niveau de qualification, et ceux vivant beaucoup à l'extérieur sont sous-représentés. Des redressements a posteriori confèrent un poids plus grand aux répondants appartenant à des catégories qui tendent à échapper à l'enquête, ils ne remédient que très partiellement à la sous-représentation des personnes les plus mobiles et/ou les plus défavorisées.

1. Les premières enquêtes Emploi du temps réalisées par l'Insee datent de 1966 et 1967. Elles ne sont pas prises en compte ici parce qu'elles ne portaient pas sur des échantillons représentatifs au plan national.

\section{Tableau A}

\section{Plans d'échantillonnage et taux de réponse des enquêtes Emploi du temps}

\begin{tabular}{|c|c|c|c|}
\hline & 1974 & 1986 & 1998 \\
\hline $\begin{array}{l}\text { Période de collecte } \\
\text { (la collecte est interrompue } \\
\text { deux semaines fin décembre } \\
\text { et deux semaines début août) }\end{array}$ & $\begin{array}{l}\text { De mai } 1974 \\
\text { à avril } 1975\end{array}$ & $\begin{array}{l}\text { De fin septembre } 1985 \\
\text { à fin septembre } 1986\end{array}$ & $\begin{array}{l}\text { De février } 1998 \\
\text { à février } 1999\end{array}$ \\
\hline Champ géographique & $\begin{array}{c}\text { Communes de } \\
2000 \text { habitants et plus }\end{array}$ & $\begin{array}{l}\text { Ensemble } \\
\text { du territoire métropolitain }\end{array}$ & $\begin{array}{c}\text { Ensemble } \\
\text { du territoire métropolitain }\end{array}$ \\
\hline Âge des répondants & 18 ans et plus & 18 ans et plus & 15 ans et plus \\
\hline $\begin{array}{l}\text { Membre(s) du ménage } \\
\text { remplissant un carnet }\end{array}$ & Kish (1) & Kish (1) et conjoint du kish & $\begin{array}{l}\text { Tous les membres } \\
\text { âgés de } 15 \text { ans ou plus }\end{array}$ \\
\hline Nombre de fiches-adresses & 10000 ménages & 16000 logements & $\begin{array}{c}12045 \text { logements, } \\
\text { dont } 10330 \text { résidences principales }\end{array}$ \\
\hline $\begin{array}{l}\text { Nombre de répondants } \\
\text { ayant fourni un carnet }\end{array}$ & 6641 & $\begin{array}{l}16047 \\
\text { (10 } 373 \text { individus-kish (1) } \\
\quad \text { et } 5674 \text { conjoints) }\end{array}$ & $\begin{array}{l}15441 \\
\text { (issus de } 8186 \text { ménages acceptant } \\
\text { l'enquête; ces ménages comptent } \\
16462 \text { membres de } 15 \text { ans ou plus) }\end{array}$ \\
\hline $\begin{array}{l}\text { Taux de réponse par rapport } \\
\text { aux fiches-adresses (en \%) }\end{array}$ & 66,4 & 64,8 & $\begin{array}{c}(8186 / 12045) \\
\times(15441 / 16462)=64,2\end{array}$ \\
\hline Taux de réponse (en \%) (2) & Non renseigné & Non renseigné & $\begin{array}{c}(8186 / 10330) \\
\times(15441 / 16462)=74,3\end{array}$ \\
\hline $\begin{array}{l}\text { Individus de } 18 \text { à } 64 \text { ans } \\
\text { vivant en zone urbaine } \\
\text { et ayant fourni un carnet }\end{array}$ & 5386 & 9975 & 8507 \\
\hline \multicolumn{4}{|c|}{$\begin{array}{l}\text { 1. (Individu-) kish : individu choisi au hasard au sein du ménage, de manière à tenir compte des inégales probabilités de sélec- } \\
\text { tion des personnes suivant la taille du ménage auquel elles appartiennent. } \\
\text { 2. Pour les enquêtes de } 1974 \text { et } 1986 \text {, les publications techniques (Insee } 1998 \text {; Insee 1990) n'indiquent pas quelle est la propor- } \\
\text { tion des résidences principales dans le total des fiches-adresses. }\end{array}$} \\
\hline
\end{tabular}


Encadré 1 (suite)

En 1974 et 1986, le codage des activités décrites en clair a été effectué manuellement. En 1998, 80 \% des activités ont été chiffrées informatiquement par une application Sicore-Emploi du temps élaborée par I'Insee (Sicore : Système informatique de codage par reconnaissance), le solde faisant l'objet d'une « reprise manuelle ».

\section{Une nomenclature d'activités largement conventionnelle}

À ces changements de procédures s'ajoute l'évolution des nomenclatures d'activités : d'une enquête à une autre, certaines des équivalences entre rubriques ne sont qu'approximatives. Le tableau B définit les correspondances retenues.

Tous les répondants ne se situent pas au même niveau de détail: certains fournissent des carnets d'une soixantaine de lignes, d'autres, d'une dizaine seulement. Si le nombre moyen de lignes était à peu près le même en 1974 et en 1986, il a sensiblement baissé en 1998 (de 27 à 20), en partie sous l'effet du passage à un pas de dix minutes : des épisodes de cinq minutes (petits trajets, travaux domestiques, conversations, etc.) qui auraient été décrits dans le cadre du dispositif de 1986 ont été omis en 1998. Le passage à un pas de dix minutes était justifié par l'inscription de l'enquête

\section{Tableau B}

Correspondances entre les nomenclatures d'activités de 1974, 1986 et 1998

\begin{tabular}{|c|c|c|c|c|}
\hline & $\begin{array}{l}\text { Nomenclature commune } \\
\text { à } 25 \text { postes }\end{array}$ & $1974(1)$ & $1986(2)$ & $1998(3)$ \\
\hline \multicolumn{5}{|c|}{$\begin{array}{l}\text { Activités professionnelles } \\
\text { et scolaires }\end{array}$} \\
\hline 1 & Travail professionnel & 11 à $15,21,22,24,25$ & 001,211 à 219, 221 à 223, 225 & 211 à 214, 221, 223, 232 à 234 \\
\hline & Études & $23,221,222$ & $\begin{array}{c}241 \text { à } 246,251 \text { à } 254, \\
261 \text { à } 263\end{array}$ & $251,252,261$ à $264,271,272$ \\
\hline \multicolumn{5}{|c|}{ Activités domestiques } \\
\hline & Cuisine, linge, ménage & $51,52,61,71,72$ & $\begin{array}{c}311 \text { à } 315,321 \text { à } 323 \\
331 \text { à } 334,345\end{array}$ & $\begin{array}{c}310 \text { à } 314,319 \text { à } 322,329, \\
330 \text { à } 332,335,339\end{array}$ \\
\hline 5 & $\begin{array}{l}\text { Soins et éducation } \\
\text { des enfants }\end{array}$ & 91 à 95, 101 à 104 & 411 à 415,421 à 425 & 410 à 414, 420 à 424 \\
\hline 6 & Courses & 112,121 à 126 & 351 à 359 & 350 à 352, 359 \\
\hline 7 & $\begin{array}{l}\text { Bricolage, jardinage, } \\
\text { soins aux animaux }\end{array}$ & 38 (hommes), 82, 83, 84 & 373 à 378 & 370 à 376,379 \\
\hline 8 & Couture & 38 (femmes), 73 & 371,372 & 333,334 \\
\hline 9 & $\begin{array}{l}\text { Travaux domestiques } \\
\text { divers }\end{array}$ & $\begin{array}{c}62,81,85,86,87,141 \\
142,201\end{array}$ & $\begin{array}{c}341 \text { à } 344,346 \text { à } 349, \\
361 \text { à } 363,379,431,432,911\end{array}$ & $\begin{array}{l}340 \text { à } 344,349,360,361,369 \\
377,419,429 \text { à } 431,439,911\end{array}$ \\
\hline \multicolumn{5}{|c|}{ Temps personnel } \\
\hline & Sommeil & 181 à 183 & 111 à 114 & 111 \\
\hline & Repas & $\begin{array}{c}161,162,171 \text { à } 174, \\
292,294,296\end{array}$ & $\begin{array}{c}141 \text { à } 147,151 \text { à } 157, \\
161 \text { à } 166\end{array}$ & 141 à 146 \\
\hline & Toilette, soins personnels & 131 à 133, 191, 192, 202 & 121 à 125,131 à $134,171,172$ & 121 à 124,131 à 133,151 \\
\hline \multicolumn{5}{|c|}{ Temps de loisir } \\
\hline & Religion, cimetière & 25 & 531 à 534,546 & 531,532 \\
\hline 14 & Associations & 261 à 268, 295, 297, 298 & 541 à 545,549 & 541,542 \\
\hline & Spectacles & 28 & 731 à 739 & 651 à 654 \\
\hline & Rencontres & 291,293 & 511 à $515,547,548$ & 510 à $513,529,543$ \\
\hline & Sport & 31 & 611 à 615 & 610 à 612 \\
\hline 18 & $\begin{array}{l}\text { Promenade, plage, pêche, } \\
\text { chasse }\end{array}$ & 321 à 325 & 621 à 627 & 620 à 625 \\
\hline & Jeux, musique & 391 à 397 & 741 à 748 & 661 à 668 \\
\hline & Conversation & $401,402,411$ & 521 à 526 & 520 à 524,533 \\
\hline & Télévision, vidéo & 361 & 714,715 & 634,635 \\
\hline 22 & Lecture & 34,35 & 711 à 713 & 631 à 633 \\
\hline & Radio, écoute de musique & 371,372 & 716,717 & 636,637 \\
\hline & Détente, pauses & $31,32,412$ & $\begin{array}{c}224,231,232,721,722, \\
921,922\end{array}$ & 231,641 \\
\hline \multicolumn{5}{|c|}{ Trajets extra-professionnels } \\
\hline & $\begin{array}{l}\text { Trajets sauf ceux à but } \\
\text { professionnel ou scolaire }\end{array}$ & $\begin{array}{c}111 \text { à } 117,151 \text { à } 157 \\
211 \text { à } 217,271 \text { à } 277 \\
301 \text { à } 307,331 \text { à } 337 \\
421 \text { à } 427\end{array}$ & 821 à 825,831 à 835,842 & $810,812,813,819$ \\
\hline \multicolumn{5}{|c|}{$\begin{array}{l}\text { 1. Codes définis dans: Huet, Lemel et Roy, Les emplois du temps des citadins, pp. 25-40, Paris, Insee, } 1978 . \\
\text { 2. Codes définis dans : Enquête sur les emplois du temps (1985-1986), fichier leda-a : tome 2, dessin de fichier, dictionnaire des } \\
\text { codes, pp. 212-215, Paris, Insee, } 1988 . \\
\text { 3. Codes définis dans : Dumontier et Pan Ke Shon, Enquête Emploi du temps 1998-1999, description des activités quotidiennes, } \\
\text { pp. 11-16, Paris, Insee, } 2000 \text {. }\end{array}$} \\
\hline
\end{tabular}


heures au cours des douze années suivantes. Pour celles en emploi, la progression, qui n'était que de 3 h 30 au cours des douze premières années, a cessé ensuite. En 1998, les personnes hors emploi disposent de 37 h 30 de loisir par semaine, celles en emploi, de 24 h 45 . L'écart s'est creusé, de six heures trente de loisir de plus pour les sans emploi en 1974, à 12 h 45 en 1998.

\section{Travail professionnel, travail domestique : une atténuation des différences entre hommes et femmes}

Observée sur la population des femmes âgées de 18 à 64 ans, la durée hebdomadaire des loisirs augmente sur les deux périodes examinées (cf. graphique I). Elle passe de 20 h 30 en 1974 à près de 25 h en 1986 et plus de 27 h en 1998 . La progression est similaire chez les hommes, qui conservent leur avantage : à chacune de ces trois dates, ils bénéficient d'un peu plus de cinq heures de loisirs de plus que les femmes.

Pour les femmes, la progression du temps libre va de pair avec une diminution de la durée du travail domestique, celle du travail professionnel restant stable, tandis que pour les hommes, elle se nourrit surtout d'une baisse du temps professionnel. Au total, les disparités entre emplois du temps féminins et masculins tendent à s'atténuer - assez rapidement de 1974 à 1986, de manière nettement ralentie ensuite (cf. encadré 2).
Chez les hommes, l'extension du chômage, l'allongement de la scolarité des jeunes et la précocité des retraites font augmenter la proportion des inoccupés (cf. tableau 2). La proportion des actifs en emploi qui atteignait $86 \%$ en 1974 passe à $73 \%$ en 1986 et à $69 \%$ en 1998. En revanche, chez les femmes, cette même proportion est stationnaire : $55 \%$ en $1974,53 \%$ en

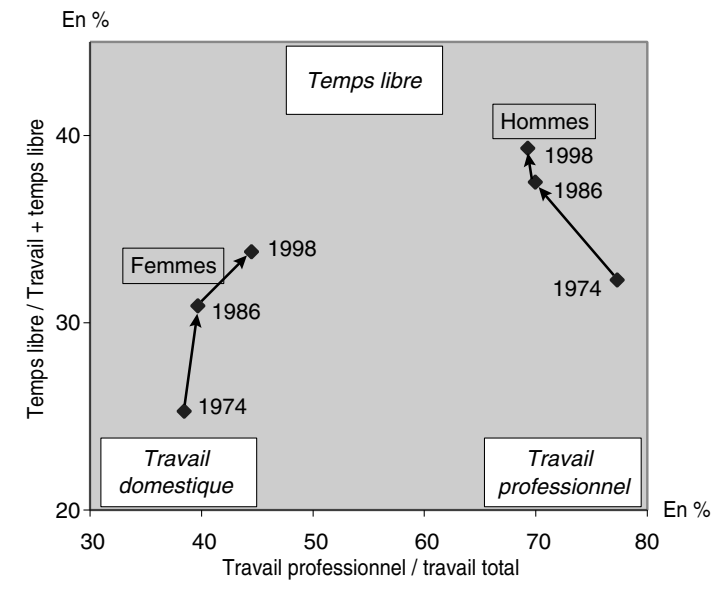

Lecture : la représentation est calquée sur Gershuny (Gershuny, 2000 , p. 132). Les flèches indiquent le sens de la chronologie. La différence entre emplois du temps féminins et masculins s'atténue sensiblement de 1974 à 1986, légèrement de 1986 à 1998. Champ : population urbaine de 18 à 64 ans.

Sources : enquêtes Emploi du temps 1974, 1986 et 1998, Insee.

Encadré 1 (fin)

française de 1998 dans un programme international coordonné par Eurostat ; les comparaisons européennes à venir ont été privilégiées au détriment de l'homogénéité de la série française.

Les activités décrites sur les carnets sont classées dans les rubriques d'une nomenclature dont les caractéristiques, largement conventionnelles, peuvent donner lieu à débat. Rendant compte de l'enquête Emploi du temps réalisée par l'Insee en 1986, Roy et Grimler regroupaient le temps passé à table et celui consacré à des relations sexuelles parmi les «activité physiologiques ». Yonnet (1999) voit là un « bel exemple d'hygiénisme militant » et ironise sur « les composantes du décidément inénarrable "temps physiologique" ". II est vrai qu'aucune classification n'est brevetée. Dans la présente étude, la nomenclature à 25 postes (cf. tableau B) s'appuie le plus possible sur les délimitations et les conventions retenues, dans le cadre d'une étude portant sur 21 pays, par le sociologue anglais Gershuny (2000), notamment pour la définition du cœur des activités domestiques (cuisine, linge, ménage). Le regroupement en cinq grands pos- tes renvoie d'abord à une opposition entre travail et consommation. Le travail total est réparti en deux composantes, le travail professionnel qui est rémunéré, et le travail domestique qui ne l'est pas. Le travail domestique (ou temps domestique) comprend des activités qui ont des équivalents marchands et qui pourraient être déléguées. À ce titre, on y fait figurer le bricolage, le jardinage et la couture - qui à d'autres égards pourraient être rangés dans le temps de loisirs. Le temps personnel et le temps de loisirs recouvrent des activités qui, par nature, ne peuvent être déléguées. Ces deux regroupements se distinguent l'une de l'autre par la récurrence des activités. Dans le temps personnel, le rythme est principalement quotidien. C'est à ce titre qu'on y classe le sommeil, les repas, l'hygiène personnelle et l'habillement. Les activités de loisir sont, elles, plus flexibles que celles du temps personnel. Un cinquième poste est constitué par les trajets autres que les parcours domicile-travail : il serait souhaitable d'imputer ce temps aux postes temps domestique, temps personnel et temps de loisirs, mais en pratique il est difficile d'opérer des distinctions fiables entre ces trois types de trajets. 


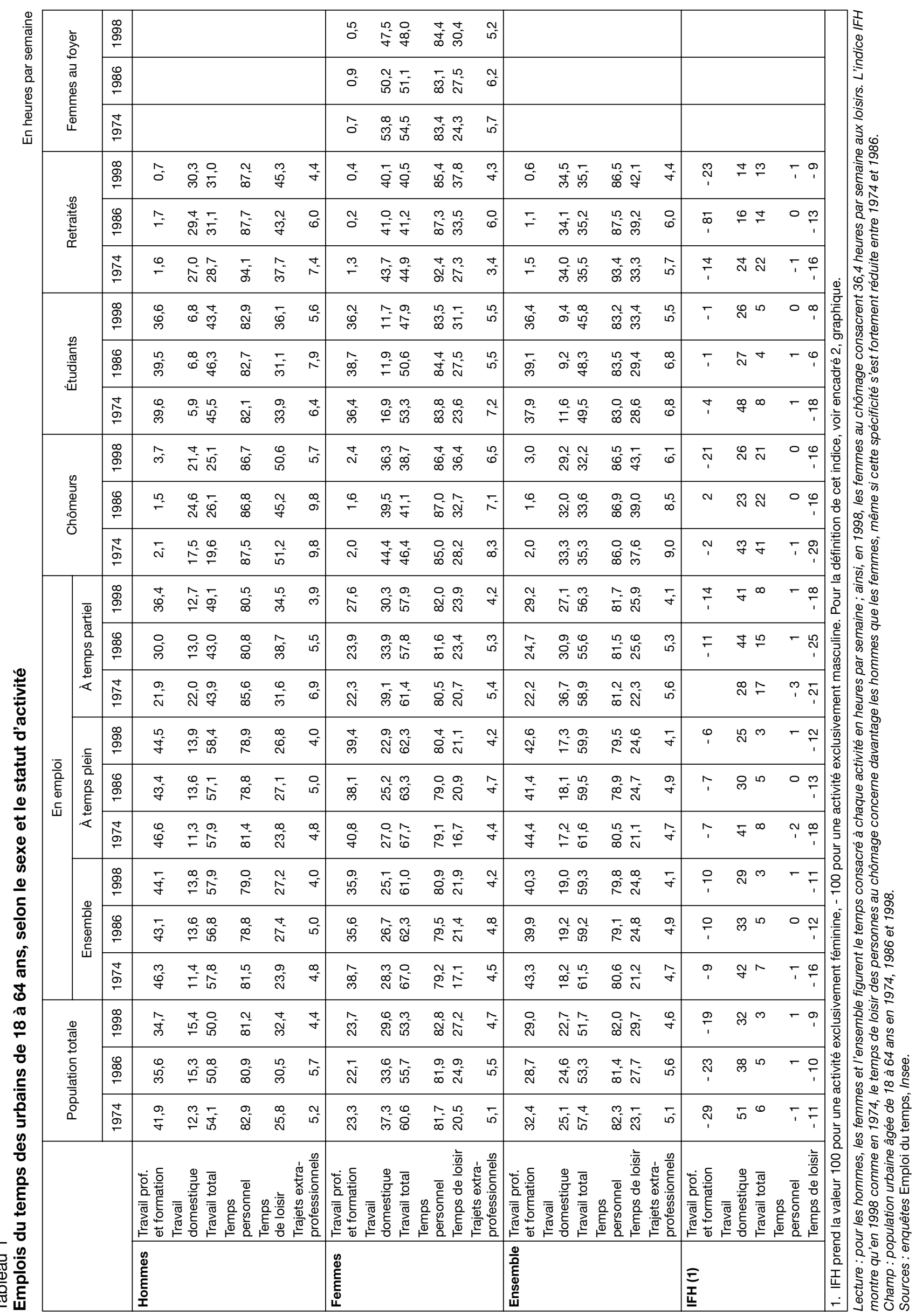




\begin{tabular}{|c|c|c|c|c|c|c|c|c|c|c|c|}
\hline \multirow{3}{*}{ 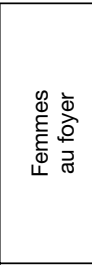 } & $\begin{array}{l}\stackrel{\infty}{\circ} \\
\stackrel{\circ}{\circ}\end{array}$ & 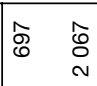 & $\stackrel{\circ}{\circ}$ & 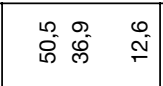 & 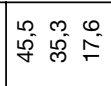 & 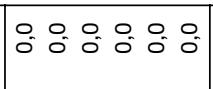 & : & : & $\therefore: 0$ & 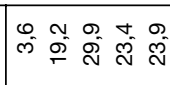 & 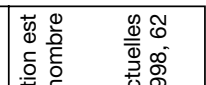 \\
\hline & $\begin{array}{l}\stackrel{\circ}{\circ} \\
\stackrel{\circ}{\leftarrow}\end{array}$ & 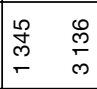 & $\stackrel{\circ}{\circ}$ & 总品 & $\mid \begin{array}{l}m \\
\bar{m} \\
\bar{m}\end{array}$ & 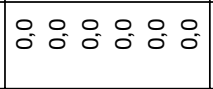 & $\therefore$ & : & $\therefore: 0$ & 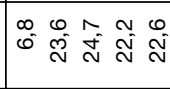 & 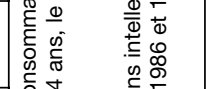 \\
\hline & 志 & 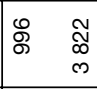 & $\stackrel{\circ}{\circ}$ & 容芯 & 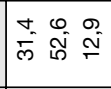 & $\therefore: 0: 00: 00$ & $\therefore$ & : & $\therefore: 0:$ & 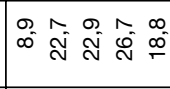 & $\begin{array}{ll}0 & 0 \\
0 & 0 \\
0 & 0 \\
0 & 0 \\
0.0 & 0 \\
0\end{array}$ \\
\hline \multirow{3}{*}{ 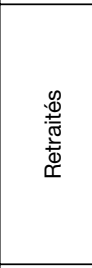 } & $\begin{array}{l}\stackrel{\circ}{\circ} \\
\stackrel{\circ}{+}\end{array}$ & 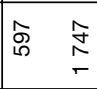 & 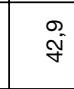 & 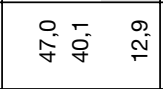 & 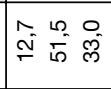 & 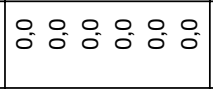 & $\therefore$ & : & $\therefore: 0$ & O: O: & 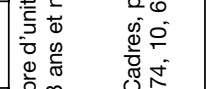 \\
\hline & $\begin{array}{l}\stackrel{\circ}{\circ} \\
\stackrel{\circ}{+}\end{array}$ & $\begin{array}{ll} & \frac{7}{6} \\
& \div\end{array}$ & $\begin{array}{l}\infty \\
\dot{q} \\
\dot{q}\end{array}$ & 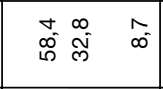 & 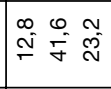 & $\therefore \circ: 00.000$. & : & : & $\therefore: 0$ & 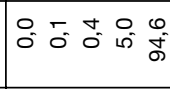 & 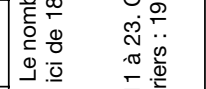 \\
\hline & 㟥 & 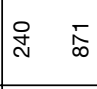 & $\begin{array}{c}\infty \\
\dot{7} \\
\end{array}$ & 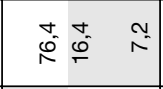 & 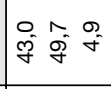 & 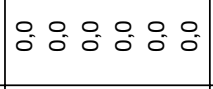 & : & : & $\therefore: 0$ & 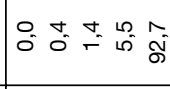 & 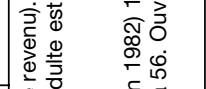 \\
\hline \multirow{3}{*}{ 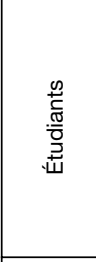 } & $\begin{array}{l}\stackrel{\infty}{\circ} \\
\stackrel{\circ}{+}\end{array}$ & $\begin{array}{ll}\hat{Q} & \stackrel{d}{N} \\
\end{array}$ & $\widehat{c}$ & 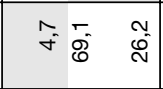 & 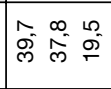 & $0_{0}^{\circ}: 0^{\circ}: 0^{\circ}: 0^{\circ}: 0^{\circ}$ & : & $:$ & $\therefore: 0$ & 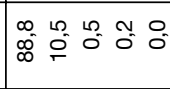 & 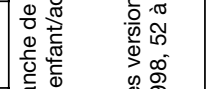 \\
\hline & $\begin{array}{l}\stackrel{\circ}{\circ} \\
\stackrel{\circ}{\circ}\end{array}$ & 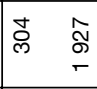 & : & 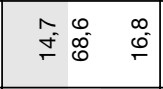 & 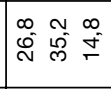 & 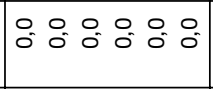 & $\therefore$ & : & $\therefore: 0$ & 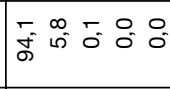 & 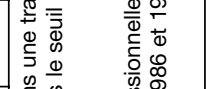 \\
\hline & 㟥 & I & $\overline{1}$ & 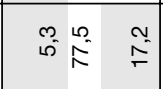 & 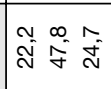 & 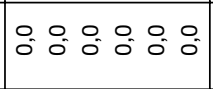 & : & : & $\therefore: 0$ & 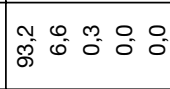 & 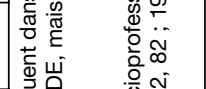 \\
\hline \multirow{3}{*}{ 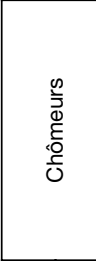 } & $\begin{array}{l}\stackrel{\infty}{\circ} \\
\stackrel{\circ}{+}\end{array}$ & 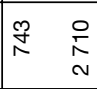 & $\hat{ن}$ & 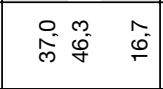 & $\begin{array}{lll}n & m & 0 \\
0 & 0 & 0 \\
0 & 0 & 0 \\
0 & 0 & 0\end{array}$ & $\because: \circ: \circ: \circ:$ & $\stackrel{\circ}{\infty}$ & 5 & 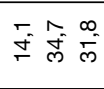 & $\begin{array}{l}m \\
m \\
\infty \\
\infty\end{array}$ & 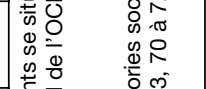 \\
\hline & $\begin{array}{l}\stackrel{\circ}{\circ} \\
\stackrel{\circ}{+}\end{array}$ & 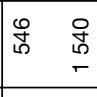 & $\begin{array}{l}\infty \\
\stackrel{q}{q}\end{array}$ & 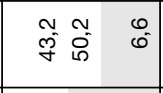 & $\frac{1}{2}=-5=$ & $\therefore: 0: 0: 0: 0$ & $\stackrel{\infty}{\infty}$ & $\bar{i}$ & 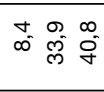 & 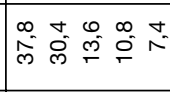 & 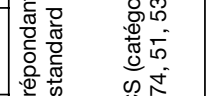 \\
\hline & 䓪 & 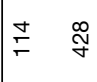 & 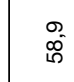 & 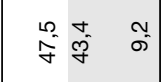 & 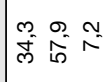 & $0_{0}^{\circ}: 0^{\circ}: 0^{\circ}: 0^{\circ}: 0^{\circ}$ & $\stackrel{\text { gi }}{\check{c}}$ & $\stackrel{\dot{\infty}}{\check{c}}$ & 它 & 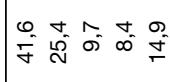 & 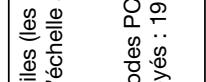 \\
\hline 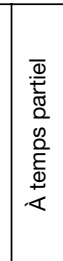 & 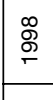 & 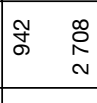 & $\frac{6}{\infty}$ & 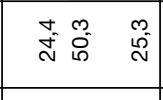 & $\hat{\sim}=\bar{c}$ & 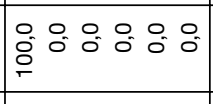 & $\stackrel{L}{\circ}^{\circ}$ & $\stackrel{\circ}{\circ}$ & 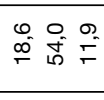 & 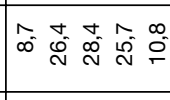 & 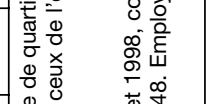 \\
\hline \multirow{8}{*}{ 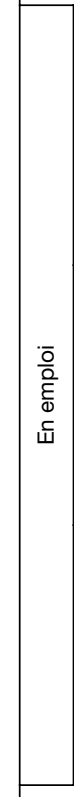 } & \begin{tabular}{|l}
$\stackrel{\circledast}{\oplus}$ \\
$\stackrel{\oplus}{\leftarrow}$ \\
\end{tabular} & 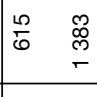 & $\begin{array}{c}\hat{\omega} \\
\infty \\
\infty\end{array}$ & 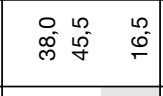 & 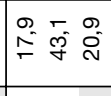 & 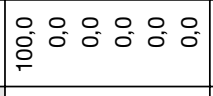 & क्? & $\stackrel{t}{\circ}$ & 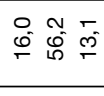 & 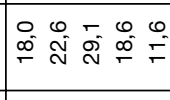 & 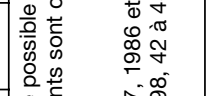 \\
\hline & 莗 & $\stackrel{\circ}{\stackrel{\infty}{N}} \frac{\vec{T}}{\infty}$ & $\begin{array}{c}n \\
\infty \\
\infty\end{array}$ & 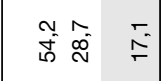 & 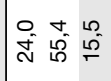 & $\begin{array}{lllll}0 & 0 & 0 & 0 & 0\end{array}$ & $\dot{\sigma}$ & $\stackrel{\infty}{\sim}$ & के & 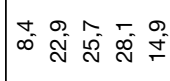 & 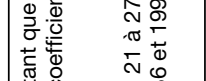 \\
\hline & $\stackrel{\stackrel{\infty}{\circ}}{+}$ & \begin{tabular}{|ll|}
\multirow{2}{*}{} & $\stackrel{8}{0}$ \\
\multirow{\gamma}{*}{} & 0 \\
\end{tabular} & $\underset{\infty}{\infty}$ & 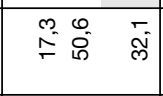 & 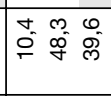 & 官 : & 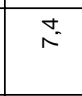 & $\begin{array}{l}\circ \\
\dot{\sigma}\end{array}$ & 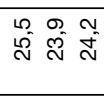 & $\overline{0}=0$ & 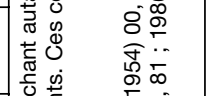 \\
\hline & 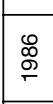 & 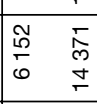 & $\begin{array}{l}\widehat{N} \\
\infty \\
\infty\end{array}$ & 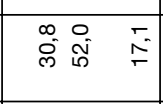 & 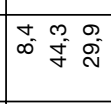 & 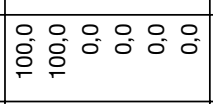 & $\begin{array}{l}\substack{\infty \\
\infty \\
\infty} \\
\infty\end{array}$ & $\hat{F}$ & 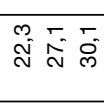 & 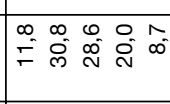 & 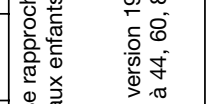 \\
\hline & 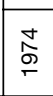 & 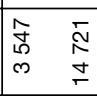 & 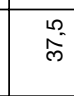 & 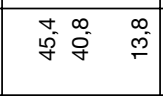 & 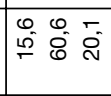 & $\begin{array}{l}0 \\
0 \\
0 \\
0\end{array}$ & $\begin{array}{l}\substack{\infty \\
\infty \\
\infty} \\
\infty\end{array}$ & $\bar{\infty}$ & 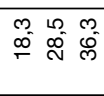 & 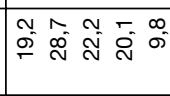 & 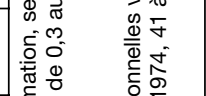 \\
\hline & $\begin{array}{ll}\infty \\
\stackrel{\infty}{\circ} \\
\stackrel{9}{\circ}\end{array}$ & 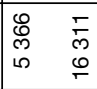 & 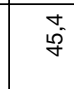 & 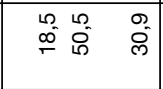 & 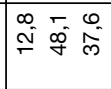 & $\begin{array}{l}0 \\
0 \\
0 \\
0\end{array}$ & $\overline{5}$ & 总 & 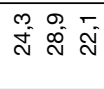 & 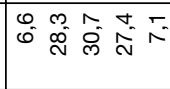 & 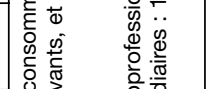 \\
\hline & \begin{tabular}{|l|}
$\stackrel{\infty}{\infty}$ \\
$\stackrel{\leftrightarrow}{\odot}$ \\
\end{tabular} & 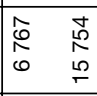 & $\begin{array}{l}\infty \\
\stackrel{\infty}{y} \\
\stackrel{y}{ }\end{array}$ & 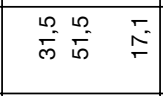 & लूr & \begin{tabular}{l}
0 \\
0 \\
0 \\
\hdashline
\end{tabular} & $\begin{array}{l}L_{0}^{\infty} \\
\infty \\
\infty\end{array}$ & $\stackrel{n}{\stackrel{\rho}{r}}$ & 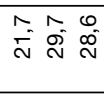 & 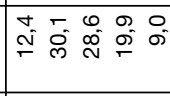 & 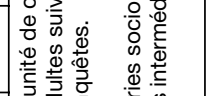 \\
\hline & \begin{tabular}{|l} 
\\
音 \\
\end{tabular} & 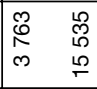 & $\begin{array}{l}0 \\
\dot{a}\end{array}$ & 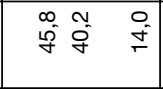 & $\begin{array}{lll}0 & m & 0 \\
0 & 0 & 0 \\
0 & 0 & 0 \\
0\end{array}$ & 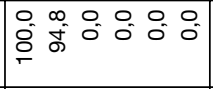 & mo & $\bar{\infty}$ & 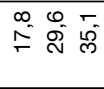 & 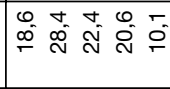 & 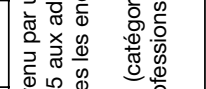 \\
\hline \multirow{3}{*}{ 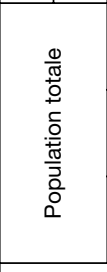 } & $\begin{array}{l}\infty \\
\stackrel{\infty}{\circ} \\
\end{array}$ & 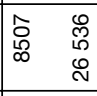 & $\frac{10}{50}$ & 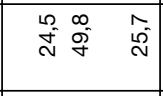 & 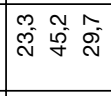 & $\mid$ & $\stackrel{\circ}{\circ}$ & $\stackrel{m}{F}$ & 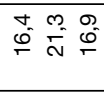 & 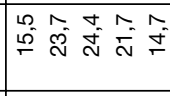 & 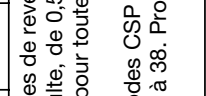 \\
\hline & $\begin{array}{l}\stackrel{\circ}{\circ} \\
\stackrel{\circ}{\leftarrow} \\
\end{array}$ & 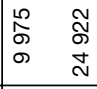 & $\begin{array}{l}\infty \\
i \\
i\end{array}$ & 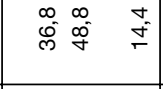 & 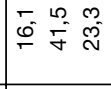 & 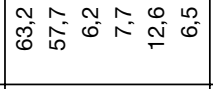 & $\mathscr{L}^{\circ}$ & 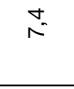 & 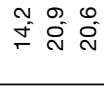 & 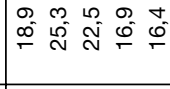 & 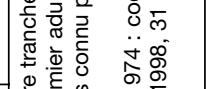 \\
\hline & 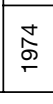 & 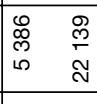 & $\bar{i}$ & 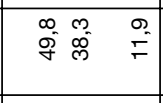 & 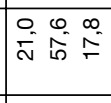 & 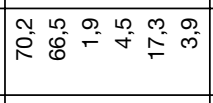 & $\begin{array}{ll}0 \\
0 \\
6\end{array}$ & $\hat{\omega}$ & 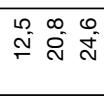 & 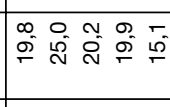 & 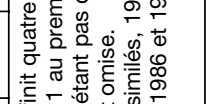 \\
\hline & & 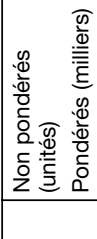 & 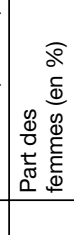 & 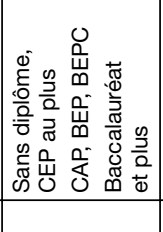 & 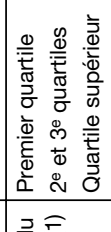 & 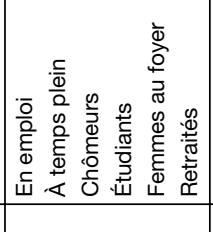 & 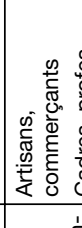 & 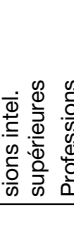 & 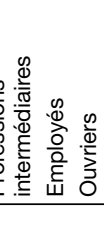 & 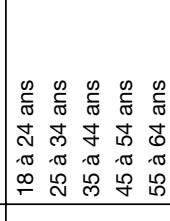 & 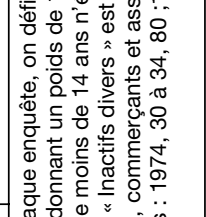 \\
\hline & & 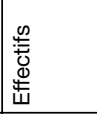 & $\begin{array}{l}\mathscr{\otimes} \\
\text { メ } \\
\infty\end{array}$ & 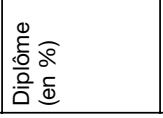 & 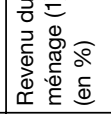 & 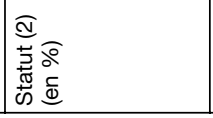 & & & & 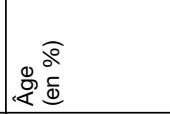 & 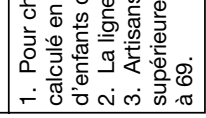 \\
\hline
\end{tabular}




\section{Encadré 2}

\section{EMPLOIS DU TEMPS FÉMININS ET MASCULINS : DISPARITÉS ET CONVERGENCES}

Les emplois du temps convergent-ils du seul fait de la modernisation du foyer ou peut-on mettre de plus en évidence le fait que les hommes accomplissent de plus en plus fréquemment des activités traditionnellement dévolues aux femmes?

Cette question conduit à examiner l'évolution des disparités homme/femme entre 1974 et 1998. On se limite à la population des personnes actives en emploi (cf. graphique). Cette population, compte tenu des exigences horaires du travail, est incitée à employer tous les moyens possibles pour gagner du temps, notamment à " externaliser » les tâches domestiques mais aussi, au sein des couples, à les répartir plus équitablement entre les conjoints.
Travail professionnel, travail domestique, temps de loisirs et temps personnel sont détaillés en 25 groupes d'activités. Au cours du dernier quart de siècle, le temps personnel est peu discriminant entre hommes et femmes. Si les hommes consacrent un peu plus de temps aux repas en 1974, cette différence s'estompe aux dates suivantes. Les activités de la toilette, l'habillement et les soins personnels, peu distinctives initialement, se féminisent légèrement en 1986 et en 1998. Enfin, aucune différence n'est constatée pour le sommeil.

Le caractère masculin du travail professionnel mais aussi des déplacements domicile/travail fait preuve d'une remarquable stabilité d'une enquête sur l'autre.

\section{Graphique}

\section{Activités féminines et masculines, en 1974 et en 1998}

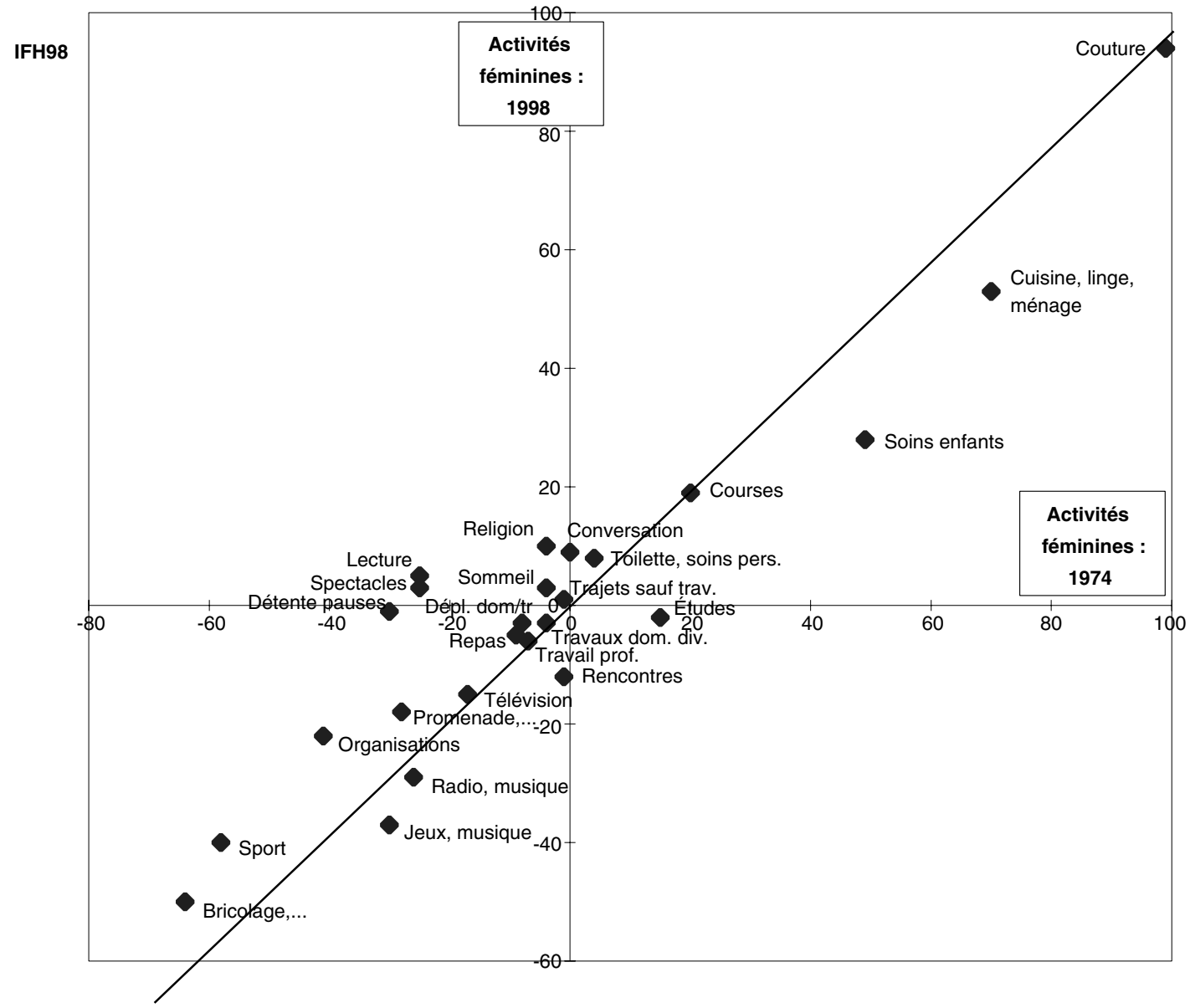

IFH74

Lecture : IFH (indicateur femme/homme) est un indicateur de la dominante masculine ou féminine d'une activité. Pour une activité de durée Df chez les femmes et Dh chez les hommes, il est défini comme suit:

$\mathrm{IFH}=200 \mathrm{Df} /(\mathrm{Df}+\mathrm{Dh})-100$

IFH prend la valeur 100 pour une activité exclusivement féminine, - 100 pour une activité exclusivement masculine, 0 pour une activité également pratiquée par les femmes et les hommes. Une valeur de 50 indique que la durée moyenne de l'activité est trois fois plus longue chez les femmes que chez les hommes.

Champ : personnes âgées de 18 à 64 ans, habitant en milieu urbain et travaillant à temps plein.

Sources : enquêtes Emploi du temps, Insee. 
1986, $54 \%$ en 1998. Les effets combinés de l'extension du chômage, de l'allongement des études et du départ à la retraite plus précoce, aussi sensibles chez les femmes que chez les hommes, sont compensés par la généralisation du travail féminin rémunéré. La part des femmes parmi les actifs ayant un emploi progresse de $40 \%$ en 1974 à $45 \%$ en 1998.

Le temps partiel contribue aussi à modérer l'ampleur de la restructuration de l'emploi du temps des actives en emploi. Si les femmes se tournent davantage vers le travail professionnel que par le passé, elles ont recours au temps partiel plus souvent que les hommes. Jusqu'en 1986, la quasi-totalité des emplois masculins sont à plein temps, $99 \%$ en 1974 et $98 \%$ en 1986 ; c'est seulement dans la dernière période qu'apparait une légère progression du travail partiel masculin (6 \% en 1998). Parmi les femmes actives, plus du dixième (11\%) étaient déjà à temps partiel en 1974. En 1998, près d'un tiers $(30 \%)$ le sont.

La durée du travail, enfin, module aussi de façon différenciée la participation des hommes et des femmes à l'emploi. À temps plein (2) les hommes travaillent cinq heures de plus, chaque semaine, que les femmes de même statut (cf. tableau 1). Aucune évolution de cet écart n'apparaît sur cette période. La baisse de la durée du travail à temps plein entre 1974 et 1986 et sa hausse au cours de la période suivante font suivre des trajectoires parallèles aux hommes et aux femmes (respectivement 46 h 36 et 40 h 48

2. Pour les actifs à temps partiel, les observations sont fragiles en raison de la faiblesse des effectifs. Compte tenu de la très faible part d'hommes à temps partiel, la durée respective du travail des hommes et des femmes qui ont adopté ce type d'emploi ne saurait remettre en cause le sens ni l'ampleur de l'écart entre hommes et femmes.

\section{Encadré 2 (suite)}

La convergence de l'emploi du temps s'observe plus nettement dans les loisirs et dans le travail domestique.

Certaines de ces activités, cependant, font exception. La couture s'affirme comme une activité quasi exclusivement féminine sur toute la période. Les courses et les démarches administratives sont, de façon permanente, plus souvent le fait des femmes. La pratique religieuse et les visites au cimetière (regroupées dans le poste religion et cimetière), s'avèrent une activité légèrement plus féminine en 1974, et cette caractéristique s'accentue aux deux dates suivantes. À l'inverse, certains loisirs domestiques - jeux et instruments de musique, radio et écoute de la musique, télévision restent aussi de façon relativement stable des activités plutôt masculines.

En matière de loisirs, la convergence vient de ce que des activités très masculines en 1974 le sont moins nettement en 1998. Le rapprochement s'effectue dès la première période pour la promenade, plage, chasse, pêche, les spectacles et sorties et surtout les sports. Ces trois groupes d'activités de loisir, qui se pratiquent presque toujours à l'extérieur du domicile, étaient le plus souvent le fait des hommes en 1974. Spectacles et sorties mettent les femmes sur un pied d'égalité avec les hommes en 1986. La période suivante, cependant, n'indique pas un renforcement de la féminisation de ce type de loisir. Le sport est devenu moins masculin en 1986 mais la tendance à la féminisation s'interrompt au cours de la période suivante. II en est de même pour la promenade, plage, pêche et chasse. Entre 1986 et 1998, l'alourdissement de la charge de travail pour les actifs en emploi n'est compensé que pour les femmes par la baisse de la durée du travail domestique. Plusieurs activités qui ont amorcé leur féminisation dans la première période continuent à se féminiser à la seconde. Détente et pauses qui occu- pent une part plus importante dans l'emploi du temps des hommes en 1974, sont presque également partagées entre les deux sexes en 1998. La lecture, activité nettement masculine en 1974, est plus féminine en 1998. La participation aux associations (parents d'élèves, syndicat, parti politique, humanitaire, etc.), fortement réservée aux hommes en 1974 et en 1986, perd une grande partie de cette spécificité en 1998.

Le poste bricolage, jardinage, soins aux animaux qui est une des composantes du travail domestique se féminise aussi de façon lente et continue sur les deux périodes. Cette évolution ne signifie pas que les femmes accroissent leur spécialisation dans les tâches domestiques, mais qu'elles la diversifient. Car pour les autres activités domestiques, la tendance est à la masculinisation. La cuisine, le linge et le ménage, d'une part, et, d'autre part, les soins et éducation des enfants opposent moins nettement l'emploi du temps des hommes et des femmes en 1986 et le rapprochement s'est accentué en 1998. Le fait que les hommes vivent plus fréquemment seuls en 1998 qu'en 1974 explique pour une part qu'ils échappent moins aux corvées domestiques. II ne vaut évidemment pas pour les soins aux enfants, auxquels les hommes prennent une part plus importante d'une enquête sur l'autre. Cette évolution ne peut être imputée à l'augmentation de la part des familles « monoparentales » (1), car dans $90 \%$ de ce type de ménage, ce sont les femmes qui ont seules la charge des enfants. Cela confirme plutôt que dans les couples avec enfants, les hommes des nouvelles générations partagent plus équitablement que dans la génération de leurs parents la responsabilité des enfants.

1. L'emploi de guillemets paraît ici nécessaire car les enfants vivant dans ce type de famille ont le plus souvent deux parents, dont l'un seulement cohabite avec eux. 
en 1974, 43 h 24 et 38 h 06 en 1986, 44 h 30 et 39 h 24 en 1998), si bien qu'en 1998 la semaine masculine est significativement plus lourde que celle des femmes.

\section{Le loisir des femmes profite du recul des tâches domestiques}

La généralisation du travail professionnel chez les femmes de 18 à 64 ans n'a pas entravé la progression des loisirs non pas en raison d'une réduction de la durée du travail à temps plein et d'une progression du temps partiel, mais du fait d'une moindre durée impartie au travail domestique.

L'essor du temps libre résulte d'un jeu de substitutions entre non pas deux mais trois ensembles d'activités, loisirs, travail rémunéré, travail domestique. Un quatrième ensemble sera négligé à ce niveau de l'analyse, le temps personnel - (sommeil, repas, toilette, habillement et hygiène personnelle). Bien que quantitativement le plus important - il occupe près de la moitié des $24 \mathrm{~h}$ journalières (cf. tableau 1 ) - ce temps est relativement incompressible, du moins dans la période et pour les populations examinées. Pour l'ensemble des 18-64 ans, chez les hommes comme chez les femmes, il occupe 82 h hebdomadaires, ses oscillations entre 1974 et 1998 n'excédant jamais une heure en plus ou en moins autour de cette valeur moyenne.

Tant sur la direction de l'évolution que sur l'écart entre hommes et femmes, il n'en est pas de même pour le travail domestique, dont la durée diminue au cours des deux périodes. La baisse est faible entre 1974 et 1986 (une demiheure) et plus accentuée ensuite (presque deux heures entre 1986 et 1998). Ces durées moyennes sont celles calculées sur la population totale des 18-64 ans des deux sexes. La faible baisse au cours de la première période résulte de deux évolutions opposées, augmentation de trois heures hebdomadaires chez les hommes et diminution de près de quatre heures chez les femmes. Au cours des 12 années suivantes, la durée du travail domestique est stationnaire chez les hommes alors qu'elle continue à diminuer chez les femmes.

Une diminution assez lente mais régulière de la durée du travail domestique féminin s'observe quel que soit le statut vis-à-vis de l'emploi. Parmi les actives à temps plein, la charge hebdomadaire passe de $27 \mathrm{~h}$ en 1974 à $23 \mathrm{~h}$ en 1998 ; parmi celles à temps partiel, de $39 \mathrm{~h}$ à $30 \mathrm{~h}$; parmi les femmes au foyer, de 54 h à 47 h 30 . Le rythme du recul est du même ordre chez les chômeuses, les étudiantes et les retraitées.

Le loisir profite du déclin des tâches domestiques. Chez les femmes inoccupées, la baisse du travail domestique se traduit directement par une progression équivalente du temps libre (cf. tableau 1). Les femmes au foyer transforment intégralement en loisirs les six heures « gagnées » sur le travail domestique. Il en est de même des huit heures que les chômeuses gagnent sur l'accomplissement des tâches du foyer. Celles qui ont un emploi à temps plein effectuent presque six heures de travail domestique en moins mais n'en convertissent que quatre en loisirs, l'augmentation du temps de travail rémunéré absorbant la différence, notamment dans la dernière période de 1986 à 1998. C'est parmi les femmes travaillant à temps partiel que le temps gagné sur le travail domestique profite le moins aux loisirs, ceux-ci n'augmentant que d'un peu plus de deux heures en un quart de siècle. Or leur temps de travail domestique a diminué, lui, de plus de huit heures trente dans la même période. Plus de six heures vont au travail rémunéré.

On a cherché à chiffrer la progression du temps de loisir et, parallèlement, la diminution de la durée du travail domestique chez les femmes en tenant compte des changements structurels qui se sont produits au cours du dernier quart de siècle, augmentation du nombre de diplômés, retard à la formation des couples, baisse du nombre d'enfants par famille, vieillissement de la population active - le pourcentage des 18-34 ans parmi les 18-64 ans diminue de $45 \%$ en 1974 à $39 \%$ en 1998 (cf. tableau 2) - et plus grande participation des femmes au travail rémunéré. La méthode statistique de la régression multiple avec variables discontinues impose de se fixer un profil de référence. On a retenu pour celui-ci, parmi les femmes, celles entre 25 et 34 ans, sans diplôme ou peu diplômées, sans enfant, vivant hors couple, habitant en immeuble collectif et actives occupées ayant un emploi à temps plein ou à temps partiel (cf. tableau 3). Observé un jour de la semaine en 1986, leur emploi du temps moyen compte 3 h 16 mn de loisir. Par rapport à ce profil, le fait de vivre en couple, ou d'avoir des enfants (toute chose égale par ailleurs, c'està-dire les autres caractéristiques demeurant inchangées) diminue le temps de loisir d'une demi-heure environ. Le temps consacré au loisir varie fortement selon que le jour observé est en semaine ou le samedi/dimanche pour les person- 
nes ayant un emploi rémunéré et selon que les personnes ont ou non un emploi rémunéré, quel que soit le jour de la semaine.

En tenant compte ainsi des principales transformations de la situation familiale et professionnelle des femmes, le temps de loisir augmente pour les femmes à l'âge actif de presqu'une heure entre 1974 et 1998 (cf. tableau 3). La progression est du même ordre de grandeur sur les deux périodes, 25 minutes entre 1974 et 1986 et 28 minutes entre 1986 et 1998 . Toutes choses égales, le temps consacré à la cuisine, au linge et au ménage diminue de 20 minutes dans chacune des deux périodes successives, la baisse du temps de travail professionnel n'étant pas significative entre 1986 et 1998 et ne comptant que pour huit minutes entre 1974 et 1986 . L'essentiel de la progression de la durée de loisir provient donc de la diminution du temps consacré aux tâches domestiques les plus lourdes et les plus traditionnellement dévolues aux femmes.
Il existe plusieurs explications à l'allégement du travail domestique des femmes et à l'augmentation concomitante de leurs loisirs. La plus couramment invoquée voit dans la baisse du travail domestique chez les femmes la conséquence d'une répartition plus égalitaire des tâches du foyer entre l'homme et la femme dans les couples. Cette explication vaut pour la première période dans la mesure où les durées de travail domestique des hommes et des femmes évoluent en sens inverse. En revanche, elle est moins recevable pour la seconde période pendant laquelle le travail domestique des hommes ne progresse plus alors que celui des femmes continue de baisser (cf. encadré 2). Une seconde explication fait référence à la modernisation du foyer et à l'accroissement du recours à des services domestiques rémunérés. L'équipement ménager, l'achat des produits prêts à consommer et la sous-traitance des tâches domestiques les plus pénibles allègent le travail domestique des membres du foyer

Tableau 3

Déterminants de la charge de travail totale, du temps de travail domestique et du temps de loisir des femmes

\begin{tabular}{|c|c|c|c|c|c|c|c|c|}
\hline & \multicolumn{2}{|c|}{ Travail total } & \multicolumn{2}{|c|}{ Travail professionnel } & \multicolumn{2}{|c|}{ Travail domestique } & \multicolumn{2}{|c|}{ Loisir } \\
\hline & Durée & Écart-type & Durée & Écart-type & Durée & Écart-type & Durée & Écart-type \\
\hline Constante & 514 & 4 & 354 & 3 & 95 & 2 & 196 & 3 \\
\hline $\begin{array}{l}1974 \\
1986 \\
1988\end{array}$ & $\begin{array}{r}30 \\
\text { Réf. }\end{array}$ & 3 & $\begin{array}{r}8 \\
\text { Réf. } \\
-5\end{array}$ & 2 & $\begin{array}{r}20 \\
\text { Réf. }\end{array}$ & 2 & $\begin{array}{l}-32 \\
\text { Réf. } \\
25\end{array}$ & 2 \\
\hline 17 à 24 ans & 28 & 4 & 9 & 3 & -68 & 2 & -12 & 3 \\
\hline $\begin{array}{l}25 \text { à } 34 \text { ans } \\
35 \text { à } 44 \text { ans } \\
45 \text { à } 54 \text { ans } \\
55 \text { à } 64 \text { ans }\end{array}$ & $\begin{array}{r}\text { Réf. } \\
22 \\
54 \\
42\end{array}$ & $\begin{array}{l}4 \\
4 \\
4\end{array}$ & $\begin{array}{r}\text { Réf. } \\
8 \\
1 \\
-4\end{array}$ & $\begin{array}{l}3 \\
3 \\
3\end{array}$ & $\begin{array}{r}\text { Réf. } \\
23 \\
39 \\
28\end{array}$ & $\begin{array}{l}2 \\
2 \\
2\end{array}$ & $\begin{array}{r}\text { Réf. } \\
-7 \\
-22 \\
-11\end{array}$ & $\begin{array}{l}3 \\
3 \\
3\end{array}$ \\
\hline $\begin{array}{l}\text { CEP, sans diplôme } \\
\text { CAP, BEP } \\
\text { Bac. et plus }\end{array}$ & $\begin{array}{r}\text { Réf. } \\
6 \\
5\end{array}$ & $\begin{array}{l}3 \\
4\end{array}$ & $\begin{array}{r}\text { Réf. } \\
4 \\
6\end{array}$ & $\begin{array}{l}2 \\
3\end{array}$ & $\begin{array}{l}\text { Réf. } \\
-25 \\
-51\end{array}$ & $\begin{array}{l}2 \\
2\end{array}$ & $\begin{array}{r}\text { Réf. } \\
7 \\
4\end{array}$ & $\begin{array}{l}2 \\
3\end{array}$ \\
\hline $\begin{array}{l}\text { Sans enfant } \\
1 \text { enfant } \\
2 \text { enfants } \\
3 \text { enf. ou plus }\end{array}$ & $\begin{array}{r}\text { Réf. } \\
47 \\
61 \\
91\end{array}$ & $\begin{array}{l}3 \\
4 \\
5\end{array}$ & $\begin{array}{r}\text { Réf. } \\
-5 \\
-18 \\
-22\end{array}$ & $\begin{array}{l}3 \\
3 \\
4\end{array}$ & $\begin{array}{r}\text { Réf. } \\
6 \\
13 \\
28\end{array}$ & $\begin{array}{l}2 \\
2 \\
3\end{array}$ & $\begin{array}{l}\text { Réf. } \\
-30 \\
-36 \\
-47\end{array}$ & $\begin{array}{l}3 \\
3 \\
4\end{array}$ \\
\hline $\begin{array}{l}\text { Seul } \\
\text { En couple }\end{array}$ & $\begin{array}{r}\text { Réf. } \\
49\end{array}$ & 3 & $\begin{array}{r}\text { Réf. } \\
-7\end{array}$ & 2 & $\begin{array}{r}\text { Réf. } \\
64\end{array}$ & 2 & $\begin{array}{l}\text { Réf. } \\
-33\end{array}$ & 2 \\
\hline $\begin{array}{l}\text { Immeuble } \\
\text { Pavillon }\end{array}$ & $\begin{array}{r}\text { Réf. } \\
10\end{array}$ & 2 & $\begin{array}{r}\text { Réf. } \\
-3\end{array}$ & 2 & $\begin{array}{r}\text { Réf. } \\
13\end{array}$ & 1 & $\begin{array}{l}\text { Réf. } \\
-15\end{array}$ & 2 \\
\hline $\begin{array}{l}\text { Temps plein ou partiel, } \\
\text { en semaine } \\
\text { Temps plein ou partiel, } \\
\text { le week- end } \\
\text { Chômage, foyer, retraite, } \\
\text { journée quelconque }\end{array}$ & $\begin{array}{r}\text { Réf. } \\
-222 \\
-215\end{array}$ & 4 & $\begin{array}{r}\text { Réf. } \\
-260 \\
-340\end{array}$ & 3 & 49 & 2 & $\begin{array}{l}\text { Réf. } \\
92\end{array}$ & 3 \\
\hline $\mathrm{R}^{2}$ & 0,35 & & 0,59 & & 0,34 & & 0,21 & \\
\hline
\end{tabular}

Lecture : régressions linéaires avec paramètres d'effet. Une femme de 25 à 34 ans, sans diplôme ou peu diplômée, sans enfant, ne vivant pas en couple, habitant un immeuble collectif, ayant un travail à temps plein ou partiel et observé un jour de semaine (du lundi au vendredi) fournissait, en 1986, 354 minutes de travail professionnel soit presque six heures par jour.

Champ : population urbaine des femmes âgées de 18 à 65 ans, soit 20410 personnes.

Source : enquêtes Emploi du temps, Insee. 
(Herpin et Verger, 2000). Cette hypothèse fait intervenir la croissance du niveau de vie et de la consommation ainsi que la diffusion, à prix décroissants, de produits qui économisent du travail domestique. Les femmes sur lesquelles retombaient l'essentiel de ces tâches tirent plus de bénéfice que les hommes de l'amélioration des conditions de vie du foyer. Mais pourquoi la durée du travail domestique qui augmente chez les hommes dans la première période ne progresse-t-elle plus dans la seconde ? L'hypothèse de la modernisation domestique n'explique pas pourquoi ces derniers fournissent au foyer un effort croissant entre 1974 et 1986 mais constant de 1986 à 1998. La dernière période voit s'amorcer une tendance nouvelle : la durée du travail professionnel augmente parmi les hommes en emploi.

\section{Les milieux populaires disposent désormais de plus de temps de loisirs...}

Les bénéfices tirés de ces évolutions en faveur du loisir n'affectent pas, non plus, à l'identique les différents milieux sociaux et professionnels (Fermanian, 1999). Sur l'ensemble de la période, la durée des loisirs augmente de dix heures, soit presque de moitié, chez les sans diplôme ou détenteurs du seul certificat d'études primaires (cf. tableau 4). Une même progression de dix heures en 24 ans s'observe parmi les titulaires de bas revenus (cf. tableau 5). Le temps libre progresse beaucoup plus lentement dans les strates supérieures, de 2 h 30 parmi ceux qui détiennent au moins le baccalauréat (cf. tableau 4) et de 3 h 30 parmi les personnes du quartile supérieur de revenu (cf. tableau 5). Au total, le gradient de la durée des loisirs en fonction de la position dans la stratification sociale s'inverse : les milieux favorisés bénéficiaient de plus de loisirs que les milieux populaires en 1974 ; c'est le contraire en 1998 (cf. graphique II). La prise en compte des congés annuels ne remet pas en cause le diagnostic (cf. encadré 3). Cette inversion est plus accentuée entre niveaux de diplôme qu'entre niveaux de revenu.

Graphique II

Effets nets du diplôme sur la durée du travail professionnel et du temps libre

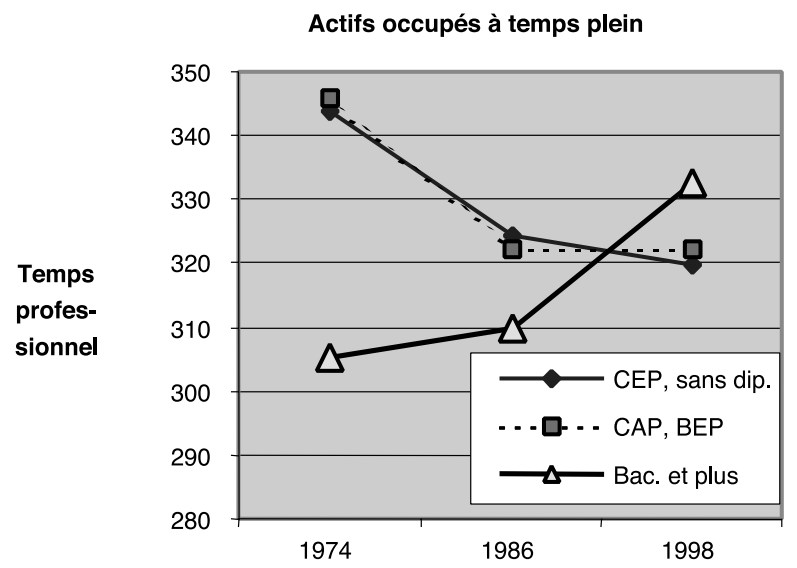

Ensemble des 18-64 ans
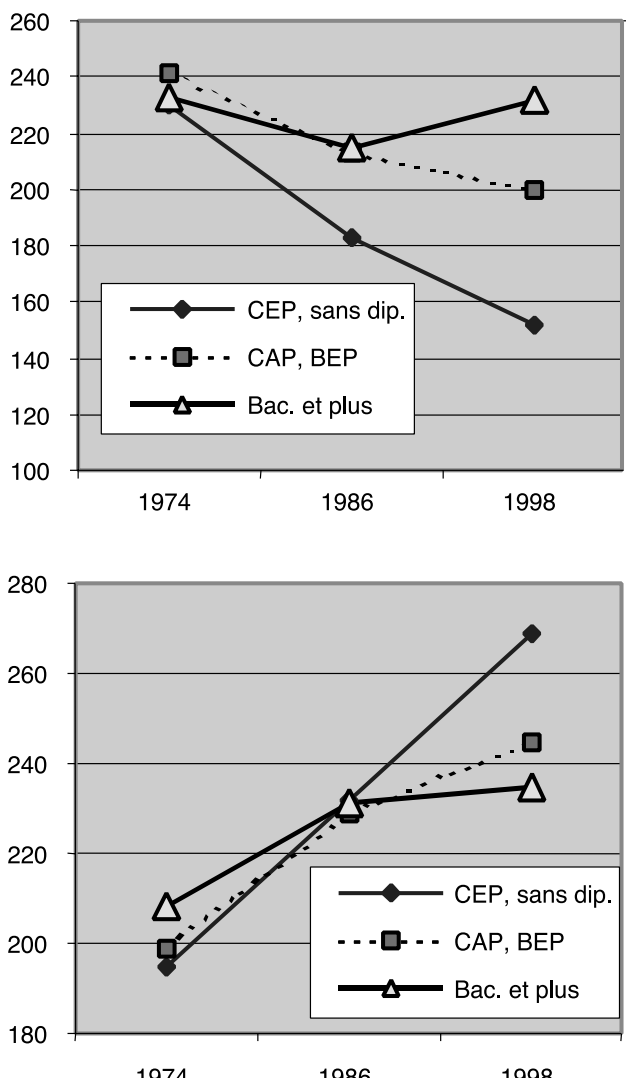

Champ : population urbaine de 18 à 64 ans. Sources : enquêtes Emploi du temps, Insee. 


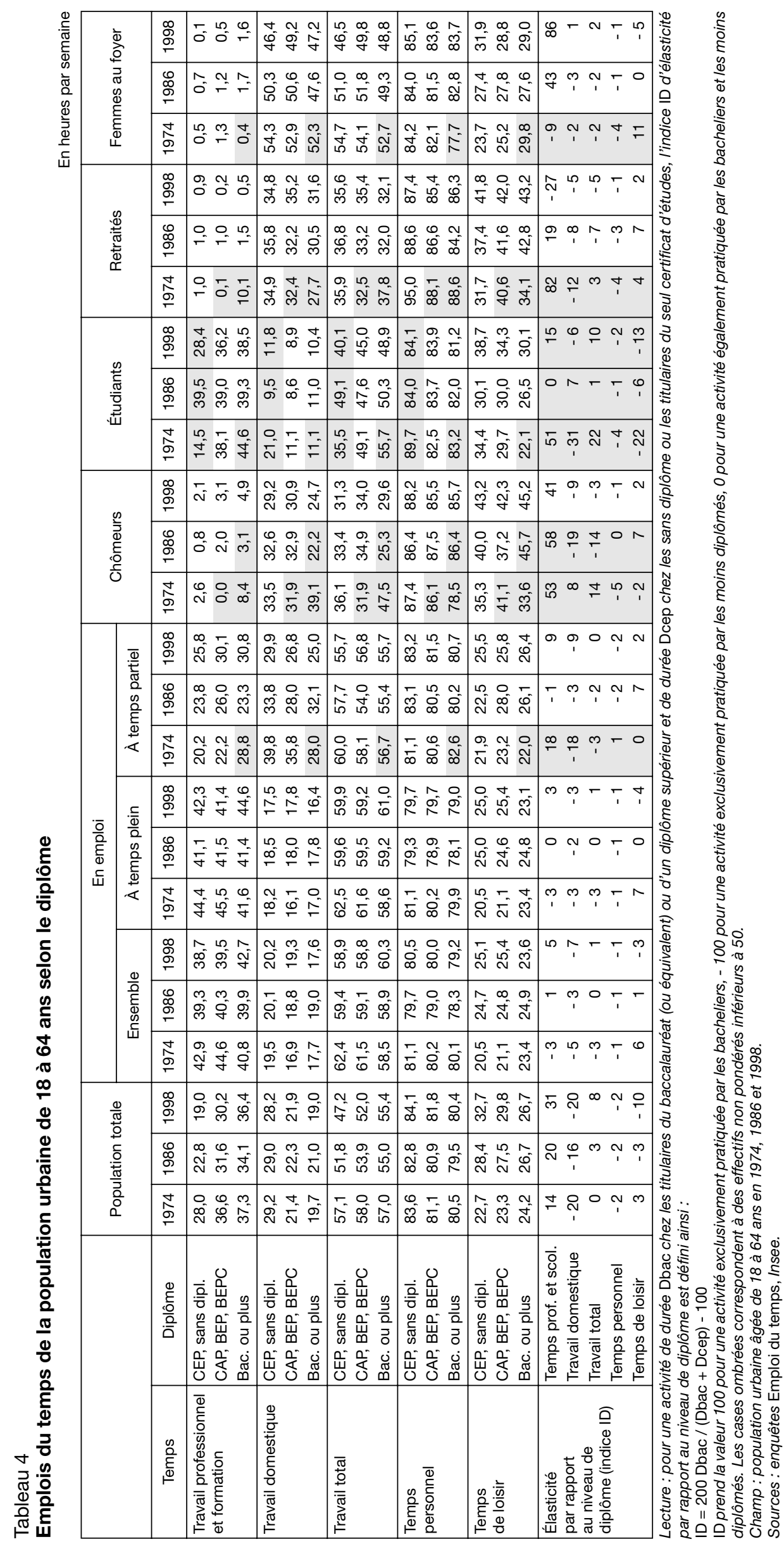




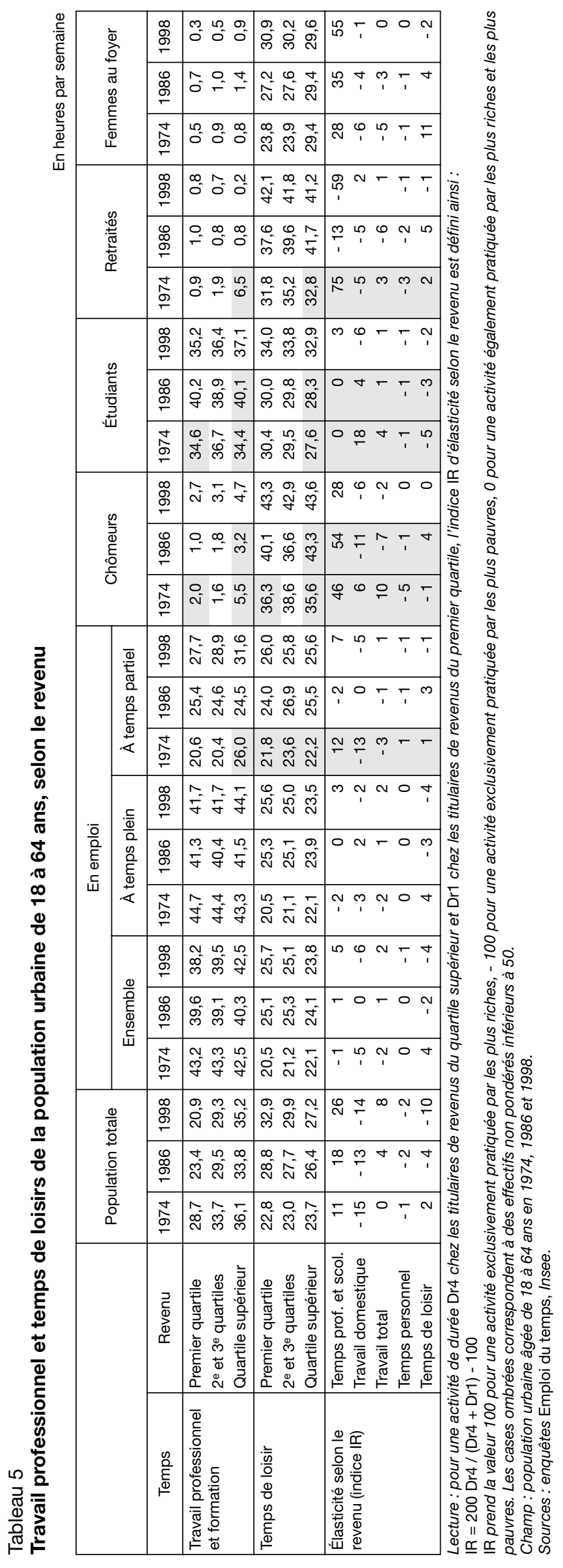




\section{... mais cette progression résulte essentiellement de l'extension du chômage}

Les milieux populaires ont-ils pour autant bénéficié d'une « libération culturelle » pour reprendre l'expression de Dumazedier ? Amère victoire, c'est d'abord par l'extension du chômage que progressent leurs loisirs (cf. tableau 5). Les chômeurs disposent en moyenne de presque deux fois plus de temps libre que les actifs en emploi (respectivement $43 \mathrm{~h} 06$ et $24 \mathrm{~h} 48$ en 1998). Or la montée du chômage a été plus sensible parmi les moins qualifiés. Entre 1974 et 1998, le taux de chômage progresse de 2,6 \% à $9,3 \%$ parmi les actifs les plus diplômés (baccalauréat et enseignement supérieur) et de 2,8 \% à

Encadré 3

\section{LES CONGÉS ANNUELS}

En 1998 et parmi la population des 18-64 ans occupant un emploi à temps plein, la corrélation est positive entre le niveau de diplôme et la durée hebdomadaire du travail professionnel. Est-ce que les plus diplômés compensent cet alourdissement par davantage de jours de congé ? L'enquête, bien que portant sur une année entière, rend insuffisamment compte des activités effectuées durant les congés puisqu'elle est interrompue à deux reprises, quinze jours au début août et quinze jours au moment des fêtes de fin d'année (cf. encadré 1). Les autres jours de congé, pris dans le reste de l'année, sont certes dans le champ de l'enquête. Cependant, ceux des ménages de l'échantillon qui sont partis en vacances et ont quitté leur domicile au moment de la visite de l'enquêteur ne sont pas interrogés. Des questions supplémentaires, portant sur les congés pris au cours des 12 derniers mois et auxquelles a répondu l'ensemble de la population active (y compris, ici, les habitants des zones rurales), permettent de juger si ces insuffisances de la collecte sont de nature à mettre en cause les résultats sur l'inversion du gradient selon le diplôme en 1998.

Si l'on met à part les enseignants (professeurs et instituteurs qui représentent un peu moins de $5 \%$ des emplois à plein temps et travaillent en moyenne 41 semaines par an), les actifs à temps plein travaillent 46 semaines et demie, soit quatre semaines de congé auxquelles s'ajoute environ une semaine et demie de fêtes fériées (Noël, jour de l'an, fête nationale, quand ces célébrations ne tombent pas le samedi ou le dimanche). Les salariés de l'État (hors enseignants) déclarent une semaine de plus que la moyenne des actifs à temps plein et les indépendants, une semaine de moins (cf. tableau A). Les salariés des grandes entreprises (plus de 500 salariés) ne bénéficient pas d'autant de congé que les salariés de l'État, mais leur dotation s'en rapproche. Symétriquement, les salariés

Tableau A

Congés annuels et durée du travail professionnel selon le statut d'emploi

\begin{tabular}{|c|c|c|c|c|c|}
\hline & \multirow{2}{*}{$\begin{array}{c}\text { Congés et } \\
\text { jours fériés (1) } \\
\text { (en semaines } \\
\text { par an) }\end{array}$} & \multicolumn{2}{|c|}{$\begin{array}{l}\text { Durée du travail professionnel } \\
\text { (en heures par semaine) }\end{array}$} & \multirow{2}{*}{$\begin{array}{c}\text { Durée annuelle } \\
\text { du travail } \\
\text { (en heures) }\end{array}$} & \multirow{2}{*}{$\begin{array}{c}\text { Répartition } \\
\text { de l'emplo } \\
\text { par statut } \\
\text { (en \%) }\end{array}$} \\
\hline & & $\begin{array}{l}\text { Journées } \\
\text { travaillées (2) }\end{array}$ & $\begin{array}{c}\text { Journées } \\
\text { faisant partie } \\
\text { des congés } \\
\text { annuels (3) }\end{array}$ & & \\
\hline Enseignants & 11,2 & 35,4 & 7,3 & 1532 & 5,3 \\
\hline $\begin{array}{l}\text { Salariés de l'État } \\
\text { (sauf enseignants) }\end{array}$ & 6,8 & 36,9 & 0,3 & 1674 & 18,3 \\
\hline Salariés des grandes entreprises & 5,9 & 39,6 & 0,0 & 1831 & 13,4 \\
\hline $\begin{array}{l}\text { Salariés d'entreprises } \\
\text { de } 10 \text { à } 499 \text { salariés }\end{array}$ & 5,5 & 40,7 & 0,4 & 1901 & 37,1 \\
\hline Salariés des petites entreprises & 5,0 & 41,2 & 2,4 & 1953 & 15,4 \\
\hline Indépendants, employeurs & 4,8 & 54,9 & 6,8 & 2630 & 10,5 \\
\hline Ensemble & 5,9 & 41,3 & 2,3 & 1920 & 100,0 \\
\hline
\end{tabular}

1. Réponses à la question «Combien de jours de congés annuels ou de semaines avez-vous effectivement pris au cours des douze derniers mois? ». Les réponses de 16 semaines ou plus ont été considérées comme formulées en jours.

2. Y compris repos hebdomadaire, congés de maladie ou de convenance personnelle.

3. Les journées observées sont des congés passés à domicile ou encadrées par des visites de l'enquêteur à la résidence principale du répondant. On suppose ici que la durée du travail est la même lorsque les congés annuels sont passés hors du domicile et à domicile.

Lecture : exemple de calcul de la durée annuelle du travail : parmi les enseignants, la durée annuelle du travail professionnel est de

$11,2 \times 7,3=82 \mathrm{~h}$ durant les congés annuels, + $(52-11,2) \times 35,4=1450 \mathrm{~h}$ durant le reste de l'année, soit au total $1674 \mathrm{~h}$.

Champ : population active urbaine âgée de 18 à 64 ans et travaillant à plein temps.

Source : Insee, enquête Emploi du Temps, 1998. 
24,9 \% parmi les CEP et sans diplôme (cf. tableau 6). La progression du temps partiel parmi les actifs en emploi, en revanche, même si elle concerne principalement les emplois peu qualifiés, n'a pas d'incidence importante sur l'accroissement de leur temps de loisir car les salariés à temps partiel, le plus souvent des femmes chargées de famille, ne consacrent pas plus de temps à leurs loisirs que les actifs employés à temps plein. La progression du chômage, par-

\section{Encadré 3 (suite)}

des entreprises employant moins de dix personnes prennent un peu plus de congés que les indépendants mais leur dotation reste inférieure à celle des autres salariés du privé.

\section{Congés annuels courts et}

lourdes semaines de travail vont de pair

Les congés annuels font l'objet d'une répartition inégalitaire mais de faible amplitude comparée à la répartition moyenne de la durée hebdomadaire du travail professionnel. Du statut d'emploi le plus favorisé sur ce plan (salarié de l'État) au moins favorisé (indépendant) l'écart est de deux semaines, soit 4,3\% quand ces deux semaines sont rapportées aux 46 semaines et demie du travail annuel moyen des emplois à temps plein. L'écart relatif est plus prononcé pour la durée hebdomadaire du temps plein. Cette moyenne est de $37 \mathrm{~h}$ parmi les salariés de l'État ( hors enseignants) et de $55 \mathrm{~h}$ chez les indépendants. Ces $18 \mathrm{~h}$ d'écart, rapportées à la durée moyenne de la semaine à temps plein, représentent une variation de $43 \%$.

Peu de jours de congé vont de pair, le reste du temps, avec une lourde semaine de travail professionnel. C'est particulièrement vrai chez les indépendants où les agriculteurs travaillent le plus longtemps en semaine ouvrée et prennent relativement moins de congés (deux semaines auxquelles on a ajouté forfaitairement les jours fériés sans pourtant que l'enquête permette d'affirmer si ces jours ont été intégralement chômés).
La même corrélation s'observe entre catégories sociales ayant des statuts d'emploi différents. Les indépendants prennent moins de congé et travaillent les jours ouvrés plus longtemps que les salariés. Parmi les salariés, ceux de l'État (hors enseignants) bénéficient à la fois de plus de jours de congé et de la semaine moyenne de travail la plus courte. Les salariés du privé occupent une situation intermédiaire, tant pour la durée des congés que pour celle de la semaine de travail.

Au sein de deux de ces trois " univers professionnels " (Singly et Thélot, 1988), la hiérarchie des emplois fait apparaître des différences faibles dans la durée annuelle des congés. Dans la Fonction publique (hors enseignants), les employés travaillent presque 37 heures par semaine et prennent cinq semaines de congé (auxquelles s'ajoutent les fêtes fériées). Les cadres travaillent deux heures de plus par semaine mais ne bénéficient que d'une demi-semaine de congé annuel supplémentaire (cf. tableau B). Dans les entreprises privées, la compensation des congés joue encore plus mal en faveur des cadres. Ces derniers ont le même nombre de semaine de congé que les membres des professions intermédiaires, alors qu'ils assurent plus de cinq heures de travail par semaine en plus. Entre cadres et employés ou entre cadres et ouvriers, la différence ne tient pas au nombre des jours de congé, mais à la durée de la semaine hebdomadaire. Dans l'entreprise privée, la dotation des cadres en jours de congé ne compense pas la lourdeur de leur charge hebdomadaire de travail.

\section{Tableau B \\ Congés annuels et durée hebdomadaire du travail selon le statut d'emploi et la catégorie socioprofessionnelle}

\begin{tabular}{|c|c|c|}
\hline & $\begin{array}{l}\text { Congés et jours fériés (1) } \\
\text { (en semaines par an) }\end{array}$ & $\begin{array}{l}\text { Durée du travail professionnel (2) } \\
\text { (en heures par semaine) }\end{array}$ \\
\hline $\begin{array}{l}\text { Salariés de l'État (sauf enseignants) } \\
\text { Cadres } \\
\text { Membres des professions intermédiaires } \\
\text { Employés }\end{array}$ & $\begin{array}{l}7,0 \\
6,8 \\
6,5\end{array}$ & $\begin{array}{l}38,7 \\
35,7 \\
36,8\end{array}$ \\
\hline $\begin{array}{l}\text { Salariés du secteur privé et semi-public } \\
\text { Cadres } \\
\text { Membres des professions intermédiaires } \\
\text { Employés } \\
\text { Ouvrier }\end{array}$ & $\begin{array}{l}5,6 \\
5,5 \\
5,1 \\
5,2\end{array}$ & $\begin{array}{l}46,1 \\
41,1 \\
37,3 \\
39,0\end{array}$ \\
\hline $\begin{array}{l}\text { Indépendants et employeurs } \\
\text { Patrons et professions libérales } \\
\text { Artisans et petits commerçants } \\
\text { Agriculteurs }\end{array}$ & $\begin{array}{l}5,4 \\
4,6 \\
3,5\end{array}$ & $\begin{array}{l}44,5 \\
55,9 \\
56,3\end{array}$ \\
\hline
\end{tabular}

Champ : population active urbaine âgée de 18 à 64 ans et travaillant à plein temps.

Source : Insee, enquête Emploi du Temps, 1998. 
ticulièrement élevée chez les hommes des milieux populaires, est la première des causes de l'inversion du gradient socio-économique du temps libre.

Les contrastes en fonction du niveau de qualification dans l'évolution de la durée du travail professionnel sont aussi moins marqués pour les actifs employés à plein temps: la durée moyenne du travail professionnel à plein temps diminue de 17 minutes par jour entre 1974 et 1986 et augmente de quatre minutes entre 1985 et 1998 .

\section{En dehors du diplôme, les déterminants sociodémographiques de la durée du travail ont peu évolué}

Ces mêmes effets ont été évalués nets des autres effets aux trois dates sur l'ensemble des 18-65 ans. Une régression multiple permet d'évaluer l'évolution de la durée du travail professionnel selon l'évolution des déterminants sociodémographiques (cf. tableau 7). À chacune des trois dates, c'est évidemment le jour observé de la semaine qui exerce la plus forte influence sur la durée de travail de la personne de référence. Le travail professionnel reste rare le dimanche - sa durée augmente légèrement de 1986 à 1998 mais diminue assez massivement et régulièrement le samedi (cf. tableau 8). Ces deux tendances opposées s'équilibrent dans l'emploi du temps : l'écart estimé entre un jour de semaine et le week-end s'avère stable d'une enquête à l'autre (cf. tableau 7). Sur la période, les écarts entre hommes et femmes sont aussi relativement stables. La durée journalière du travail professionnel des premiers excède de 38 à 45 minutes celle du travail professionnel des femmes. Les effets d'âge sont de plus faible ampleur et, en général, l'écart à la modalité de référence n'est pas significatif. Il en est de même si la personne vit ou non en couple. Le

Tableau 6

Taux de chômage selon le niveau de diplôme

\begin{tabular}{|l|c|r|c|}
\hline \multicolumn{1}{l|}{} & 1974 & 1986 & 1998 \\
\hline Sans diplôme, CEP au plus & 2,8 & 11,8 & 24,9 \\
CAP, BEP, BEPC & 2,7 & 9,2 & 13,9 \\
Baccalauréat et plus & 2,6 & 5,2 & 9,3 \\
\hline Ensemble & 2,7 & 8,9 & 14,2 \\
\hline
\end{tabular}

Lecture : dans l'enquête Emploi du temps, un chômeur est défini comme une personne sans emploi et déclarant en chercher un. Champ : population urbaine âgée de 18 à 64 ans en 1974, 1986 et 1998.

Sources : enquêtes Emploi du temps, Insee. nombre d'enfants dans le ménage est lié plutôt négativement à la durée du travail (3). L'habitat - maison individuelle ou logement en immeuble collectif - n'a pas d'incidence sur la durée du travail (la taille de l'agglomération de résidence n'est pas introduite dans cette régression) (cf. tableau 8 et encadré 4). Ces six variables du modèle, quand on leur ajoute le diplôme, expliquent presque les deux tiers de la variance observée de la durée du travail à chacune des trois dates. Les rôles du sexe, du nombre d'enfants et du jour de la semaine sont d'ampleur analogue et de même sens à chaque date. Il n'en est pas de même pour le niveau de diplôme.

\section{Ce sont maintenant les plus diplômés qui travaillent le plus}

En 1974, les détenteurs du bac et de diplômes plus élevés travaillent professionnellement moins (24 minutes de moins par jour) que ceux sans diplôme ou n'ayant obtenu que le certificat d'études primaires (cf. tableau 7). Les titulaires de diplômes intermédiaires (CAP et BEP) ont alors une durée du travail proche de celle des moins diplômés. Dans l'enquête de 1986, les plus diplômés ne se distinguent plus des moins diplômés et des diplômés moyens par la durée du travail. Dans la dernière enquête, en 1998, les variations liées au diplôme se sont inversées. Les détenteurs de diplômes moyens travaillent huit minutes par jour de plus que les moins diplômés. Les détenteurs du baccalauréat et de diplômes supérieurs les devancent de 19 minutes par jour. Cette évolution contrastée de la durée du travail selon le niveau de diplôme concerne les hommes comme les femmes (4). Désormais, ce sont les plus diplômés qui travaillent relativement plus longtemps à l'âge actif.

L'inversion du gradient selon le diplôme se constate aussi pour la durée du loisir, toutes choses égales par ailleurs (cf. tableau 7). Parmi les 18-64 ans, l'écart net était à l'avantage des plus diplômés en 1974 (17 minutes de plus par jour). Cet avantage disparaît en 1986. Les plus diplômés sont pénalisés dans leurs loisirs de 14 minutes en 1998 par rapport aux sans diplôme et peu diplômés. Contrairement aux pronostics de Dumazedier, on n'assiste pas à une extension générale du temps de loisirs, mais à un déplacement de la charge de travail vers les

3. Cette corrélation négative qui n'est significative ni en 1974 ni en 1986, l'est cependant en 1998.

4. Les régressions séparées selon le sexe, qui ne figurent pas dans l'article, peuvent être consultées auprès des auteurs. 


\begin{tabular}{|c|c|c|c|c|c|c|c|c|c|c|c|c|c|c|c|c|}
\hline \multirow{6}{*}{$\frac{\frac{\rho}{0}}{\frac{0}{9}}$} & 吅 & 音 & o & $\sim$ & $\theta \quad \partial \theta \partial$ & $m \sigma$ & 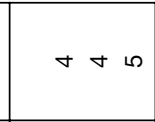 & $m$ & $m$ & & & $\nabla$ & & & & 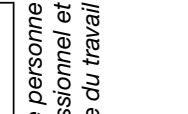 \\
\hline & & 邑 & ¿্ণ & 希 & 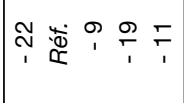 & 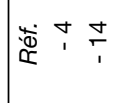 & 岕 & 范 & 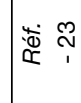 & & & $\stackrel{N}{N}$ & $\stackrel{\leftrightarrow}{\stackrel{\circ}{\sim}}$ & 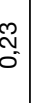 & & 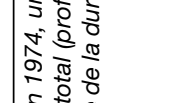 \\
\hline & 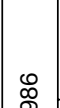 & 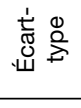 & \% & $\sim$ & $\infty \quad m+\gamma$ & $n+$ & $m \infty$ & $m$ & $\sim$ & & & m & m & & & 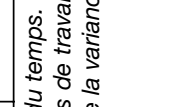 \\
\hline & & 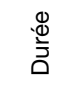 & $\stackrel{\text { N }}{\text { N }}$ & 4 & 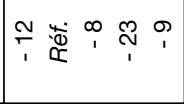 & 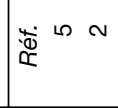 & 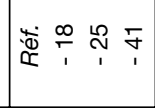 & 离 & 幽 & & 若 & $乏$ & $\stackrel{\mathbb{N}}{\sim}$ & ב্ & & 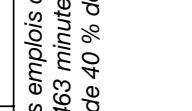 \\
\hline & & 章 & is & $m$ & م & m & $\nabla \infty 0$ & $\nabla$ & $m$ & & & $\nabla$ & $\diamond$ & & & 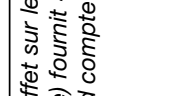 \\
\hline & $\stackrel{9}{\tau}$ & 离 & $\stackrel{\leftrightarrow}{\circ}$ & 这? & 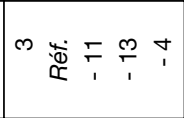 & 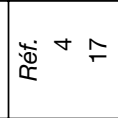 & 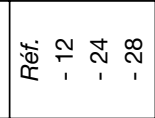 & 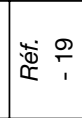 & 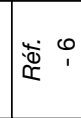 & & & $\stackrel{\infty}{\rightleftharpoons}$ & $\stackrel{\widetilde{m}}{\sim}$ & ר్ & & 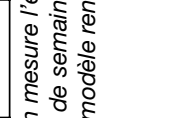 \\
\hline \multirow{6}{*}{ 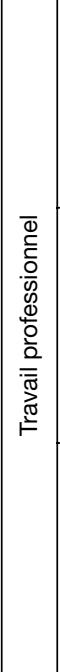 } & $\stackrel{\infty}{8}$ & 窇 & \% & $\sim$ & $\theta \quad \theta \theta$ & $m+$ & $m+\infty$ & $m$ & $\sim$ & & & $\nabla$ & m & & & 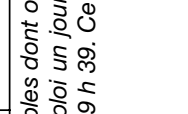 \\
\hline & & $\stackrel{\oplus}{\stackrel{\oplus}{\bar{\Delta}}}$ & প্ল & 若 & $0 \underset{\mathbb{\infty}}{0} 0$ & 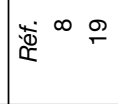 & 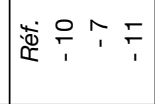 & $\underset{4}{4}$ & 岸 & & & 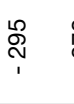 & 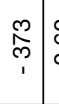 & $\stackrel{8}{\circ}$ & & 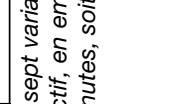 \\
\hline & œ & 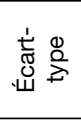 & o & $\sim$ & $\infty \quad \forall \forall+$ & $\sim \sigma$ & $m \infty$ & m & $\sim$ & & & n & m & & & 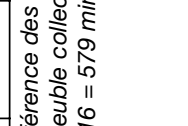 \\
\hline & & 离 & 亲 & 苞早 & 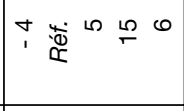 & 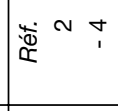 & 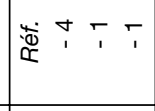 & יָ & 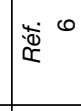 & & 苂 & న్ & $\begin{array}{ll}\hat{o} \\
\text { p } \\
1\end{array}$ & E. & & 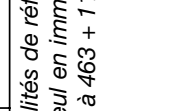 \\
\hline & $\frac{9}{4}$ & 音 & 0 & $\diamond$ & $0 \quad 000$ & +0 & م 1 & م & $\nabla$ & & & ص. & $\checkmark$ & & & 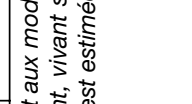 \\
\hline & & 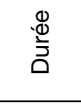 & $\stackrel{\infty}{\sim}$ & 范 守 & 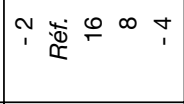 & 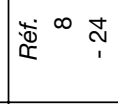 & $\begin{array}{llll} & 0 & 0 & 0 \\
0 & 1 & 1 & 1\end{array}$ & i & $\stackrel{\sim}{\pi}_{\infty}^{\infty}$ & & 若 & 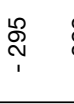 & ळ్ & : & & 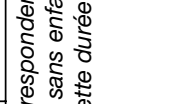 \\
\hline \multirow{6}{*}{ 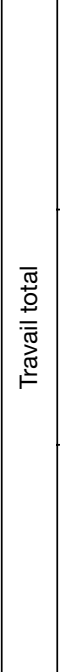 } & \multirow{4}{*}{$\begin{array}{l}\stackrel{\circ}{\circ} \\
\stackrel{\circ}{\leftarrow}\end{array}$} & 紊 & $\infty$ & $m$ & $\infty \quad \infty+\infty$ & $m+$ & $+\infty 0$ & 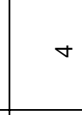 & m & & & ص. & $\diamond$ & & & 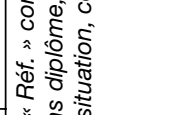 \\
\hline & & 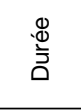 & $\underset{f}{\frac{1}{N}}$ & 若离 & 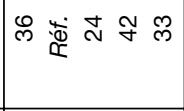 & 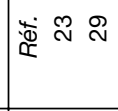 & 岗 & 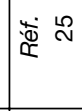 & 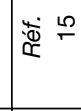 & & 芯 & 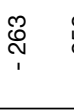 & \begin{tabular}{c|c}
$\stackrel{\infty}{\stackrel{N}{N}}$ \\
1 & $\vdots$ \\
1 &
\end{tabular} & c. & & 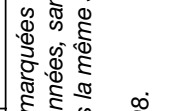 \\
\hline & & 紊 & $\infty$ & $m$ & o $\quad \nabla \omega \omega$ & $\infty 6$ & 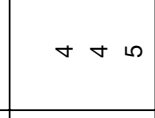 & $m$ & $m$ & & & $\diamond$ & m & & & 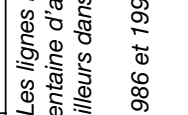 \\
\hline & & 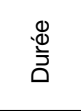 & P & 舫 & 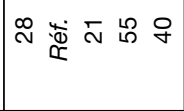 & 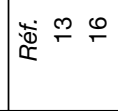 & 菦 チ & 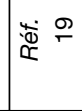 & 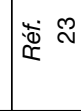 & & 苂 & 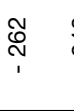 & 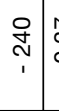 & o. & & 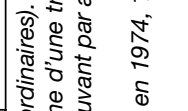 \\
\hline & \multirow{2}{*}{ 离 } & 站 & o & 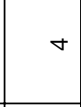 & $\wedge \quad 00 \Omega$ & $\curvearrowleft \wedge$ & $0 \wedge \infty$ & م & 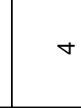 & & & $\circ$ & & & & 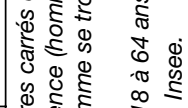 \\
\hline & & $\stackrel{\mathscr{D}}{\stackrel{\mathscr{I}}{0}}$ & ஜֶ & 若豙是 & @ & 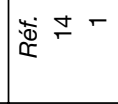 & 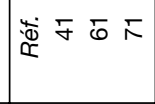 & 若学 & $\ddot{\mathbb{\alpha}} \sim$ & & 苂 & $\stackrel{\stackrel{\leftrightarrow}{N}}{1}$ & 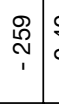 & $\begin{array}{c}9 \\
0 \\
0\end{array}$ & \&ิ & 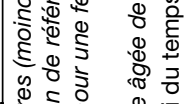 \\
\hline & & & 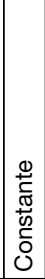 & 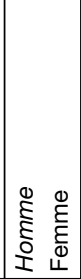 & 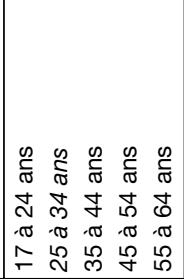 & 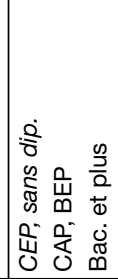 & 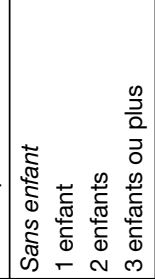 & 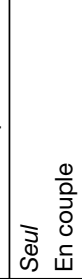 & 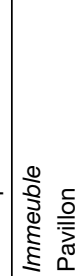 & 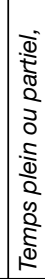 & 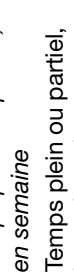 & 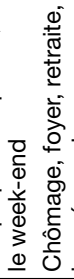 & $\begin{array}{l}0 \\
0 \\
0 \\
0 \\
0 \\
0 \\
0 \\
0 \\
0 \\
0 \\
0 \\
0 \\
0 \\
0 \\
0\end{array}$ & & & 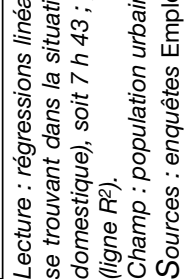 \\
\hline
\end{tabular}


catégories sociales les plus qualifiées. Ce déplacement se traduit par deux évolutions se renforçant l'une l'autre. La structure des emplois s'est transformée, les emplois peu qualifiés diminuant en nombre et les plus qualifiés se multipliant. De plus, la durée du travail fourni par les moins qualifiés a diminué, tandis qu'elle a légèrement augmenté chez les plus diplômés, toutes choses égales d'ailleurs et notamment une fois contrôlée l'incidence du chômage.

\section{Le « bonheur au travail » des cadres}

Dans l'entreprise, les horaires des cadres sont moins strictement délimités que ceux des autres salariés. L'allongement de leur temps de travail a pu faire l'objet de pressions d'autant plus fortes que la main-d'œuvre très qualifiée est demeurée rare. Cette explication par la politique des employeurs ne suffit pas à expliquer sa réussite. D'où vient que l'alourdissement de la charge du travail des plus diplômés ne rencontre pas plus de résistance de leur part ? Plusieurs explications ont été envisagées. La première est celle de l'augmentation du revenu tiré du travail. Les diplômés travaillent davantage, mais ont vu leur situation salariale s'améliorer en proportion. Dans des pays tels que les ÉtatsUnis et le Royaume-Uni, cette explication est sans doute recevable (Atkinson et al., 2001). Elle l'est moins en France, où n'apparaît pas d'accroissement important des inégalités salariales en faveur des plus qualifiés au cours de la

Encadré 4

\section{L'EMPLOI DU TEMPS DES RURAUX}

L'enquête Emploi du temps de 1986 et celle de 1998 couvrent l'ensemble de la population française, rurale comme urbaine. Celle de 1974 ne porte que sur les unités urbaines de 2000 habitants et plus (cf. encadré 1). La comparaison aux trois dates impose donc qu'on se limite à la population urbaine. Les tendances que cet article fait ressortir seraient-elles très différentes si le champ de l'étude était élargi à l'habitat rural ? Cet examen est entrepris pour les deux dernières enquêtes où les ruraux sont représentés.

L'emploi du temps des ruraux âgés de 18 à 64 ans présente des caractéristiques moyennes qui les différencient des urbains des mêmes tranches d'âge. En 1986, les premiers consacrent trois heures de plus par semaine que les urbains au travail domestique, une heure de plus au temps personnel, une demi-heure de plus au travail professionnel. En contrepartie, les déplacements domestiques et de loisirs (une heure de moins que les urbains) et les loisirs (plus de trois heures en moins que les urbains) tiennent moins de place dans leur emploi du temps. Ces différences observées sont brutes. Elles s'expliquent en partie parce que les deux populations n'ont pas les mêmes caractéristiques démographiques ni le même mode de vie. La population des 18-64 ans est plus âgée en milieu rural : $22 \%$ ont entre 50 et 64 ans contre seulement $10 \%$ en milieu urbain. Les activités de bricolage et de jardinage, classées parmi le travail domestique, sont plus fréquentes dans la maison individuelle que dans l'appartement d'un immeuble collectif. Or la maison individuelle est le mode d'habitat par excellence des zones rurales : $94 \%$ des résidences principales contre $46 \%$ seulement dans les villes et leurs agglomérations.

\section{Des évolutions analogues} à la campagne et à la ville

Les évolutions, en revanche, sont analogues à celles établies sur la population des seuls urbains. Entre
1986 et 1998 , le temps personnel est en légère croissance, de 48 minutes par semaine, pour les ruraux comme pour les urbains. Le travail domestique diminue de deux heures pour les urbains et d'une heure et demie pour les ruraux. Un rapprochement s'esquisse pour les loisirs : ces activités augmentent de deux heures pour les urbains et de deux heures trente pour les ruraux. Le travail professionnel se caractérise, comme les niveaux de formation dans le cas de la population urbaine, par une inversion du gradient. Les actifs travaillent légèrement plus longtemps à la campagne qu'à la ville en 1986 (29 h 16 contre 28 h 42 en 1986) ; c'est l'inverse en 1998 ( 27 h 36 contre 29 h). Ces évolutions sont établies toutes caractéristiques confondues. La baisse de la durée de travail des habitants des campagnes pourrait résulter de l'extension du chômage et des autres formes d'inoccupation (allongement de la scolarité des jeunes, extension des préretraites) : ce n'est manifestement pas le cas, dans la mesure où la part d'actifs occupés n'a pas fléchi en milieu rural : $64 \%$ en 1986 et $65 \%$ en 1998. Le recul de la durée du travail professionnel dans ces zones provient pour une part du déclin relatif des indépendants (19\% en 1986 et $11 \%$ en 1998). En effet, leur journée de travail est en moyenne plus longue que celle des salariés, y compris les cadres, en 1998 (cf. encadré 3). Elle s'explique également par l'importance croissante prise par les employés (13\% en 1986, $18 \%$ en 1998), qui travaillent plus souvent à temps partiel que les autres catégories. Bien que l'on compte encore en 1998 moins de cadres à la campagne qu'à la ville, la structure sociale de la population rurale s'est rapprochée de celle des villes.

Substitution des loisirs au travail domestique, stabilité du temps personnel et inversion du gradient de qualification dans le travail professionnel, le diagnostic sur l'évolution de l'emploi du temps ne semble donc pas remis en cause par l'évolutions de l'emploi du temps de la population rurale entre les deux dernières enquêtes. 
période étudiée (Fougère et Kramarz, 2001). La seconde hypothèse a trait à la mobilité ascendante. La structure de l'emploi se déformant vers le haut, la société française a connu une période favorable aux trajectoires ascendantes, mobilité en cours de carrière et mobilité entre générations confondues (Vallet, 1999). Les titulaires des emplois qualifiés seraient plus nombreux que par le passé à percevoir comme réussie (ou pleine de promesses) la carrière qu'ils suivent et seraient donc motivés à en faire un peu plus. La troisième hypothèse a trait aux conditions et la nature du travail : le « bonheur au travail », l'implication positive et passionnelle dans le travail s'observent plus fréquemment parmi les titulaires d'emplois très qualifiés, plus rarement chez les ouvriers et les employés (Godechot et al.,1999; Baudelot et Gollac, 2001). Les initiatives éventuelles des cadres font l'objet, le plus souvent, d'une « reconnaissance » par leurs instances de contrôle ou leurs hiérarchies. Le travail est aussi moins solitaire et consiste plus souvent en activités collégiales. L'évolution vers des formes plus individualisées de négociation des conditions de travail entre salariés et employeurs favoriserait un plus grand intérêt pour le travail. Quand le travail devient plus intéressant, l'attirance pour les loisirs décroît. Lorsqu'il s'accompagne d'une meilleure rémunération, il permet d'accéder à des loisirs nouveaux pour la personne, ou d'éliminer les temps morts et les aspects les plus pénibles des loisirs habituels : l'adhésion à un cercle huppé permet au sportif d'accéder à des équipements de haute qualité, grâce à un matériel sophistiqué un amateur de voile limite le temps consacré à une opération fastidieuse telle que le gréement, le recours à des moyens de transports coûteux permet d'accéder rapidement et confortablement aux lieux de pratique, etc. Le temps de loisir peut alors diminuer sans qu'il en

Tableau 8

Durée quotidienne du travail quotidien selon le jour de la semaine

\begin{tabular}{|c|c|c|c|c|}
\hline & 1974 (1) & 1986 (1) & 1998 (1) & $\begin{array}{c}\text { Variation } \\
\text { 1974-1998 (2) }\end{array}$ \\
\hline $\begin{array}{l}\text { Lundi } \\
\text { à vendredi }\end{array}$ & 7h 07 & 6 h 50 & 6 h 56 & -11 \\
\hline Samedi & 2 h 59 & 2 h 42 & $2 \mathrm{~h} 16$ & -43 \\
\hline Dimanche & 0 h 58 & 0 h 51 & $1 \mathrm{~h} 04$ & +6 \\
\hline
\end{tabular}

Lecture : en 1974, la durée moyenne de travail du lundi au vendredi est de 7 h 07 par jour.

Champ : population urbaine âgée de 18 à 64 ans en 1974, 1986 et 1998.

Sources : enquêtes Emploi du temps, Insee. aille de même pour la satisfaction que l'on en tire (Linder, 1990).

Aucune de ces trois explications ne peut être pleinement mise à l'épreuve. Car la série des enquêtes Emploi du temps ne fournit pas les informations qui seraient nécessaires pour mesurer la portée et les limites de chacune d'entre elles. Sauf pour la dernière hypothèse. Dans l'approche de Linder, en effet, les loisirs sont supposés en concurrence les uns avec les autres. Est-ce que parmi les titulaires d'emplois qualifiés le retour à une semaine de travail plus longue mais aussi mieux rémunérée n'entraîne pas des arbitrages défavorables à la lecture et à la télévision qui sont des loisirs à la fois consommateurs de temps et peu susceptibles d'être améliorés par le recours à des services marchands?

L'arbitrage entre activités de loisir sous la contrainte budgétaire est plus impératif pour les 18-64 ans en emploi que pour ceux qui sont inactifs ou au chômage. Aussi limite-t-on le champ de l'analyse à la population active occupée. On privilégie le diplôme en tant qu'indicateur de différenciation sociale : les variations de la durée des activités en fonction du niveau de revenu sont dans l'ensemble un peu moins amples (cf. tableaux 4 et 5) et celles selon la catégorie socioprofessionnelle impliqueraient de trop longs développements. Les activités peuvent être ordonnées selon un indice caractérisant leur sous-représentation - ou leur surreprésentation - parmi les plus diplômés (Indice ID, cf. tableau 4 et graphique III). Les activités qui se situent au-dessus de la diagonale ascendante du graphique III sont celles dont la durée moyenne, de 1974 à 1998, s'est accrue plus vite (ou a diminué plus lentement) parmi les bacheliers que parmi les sans diplôme, l'importance relative, chez les non diplômés, de celles qui sont au-dessous s'est au contraire affirmée.

Les repas, le sommeil, la toilette et les soins personnels, les courses, les soins et l'éducation des enfants sont, tout au long du quart de siècle observé, des activités pratiquées de manière à peu près uniforme quel que soit le niveau de diplôme : elles sont socialement peu discriminantes.

\section{La détermination des loisirs par le niveau de formation s'est accentuée}

Les activités caractéristiques, en 1974 et en 1998, des personnes les moins diplômées - et 
donc des milieux populaires - sont la couture, la télévision, le bricolage, les travaux domestiques de cuisine, d'entretien du linge et de ménage. La sur-représentation, parmi ces personnes, de la couture, du bricolage et du temps consacré à la télévision s'est accrue entre ces deux dates. En 1974, les actifs occupés titulaires du certificat d'études ou sans diplôme consacraient chaque jour 1 h 18 à la télévision, en 1998 ils sont passés à 2 h 02 ; les bacheliers et les diplômés de l'enseignement supérieur passent, eux, de 54 minutes à $1 \mathrm{~h} 10$.

Inversement, les activités les plus caractéristiques des titulaires de diplômes élevés sont, en 1998 comme en 1974, en dehors des études elles-mêmes, la participation à la vie associative et la fréquentation des spectacles. La lecture et la pratique des jeux ou de la musique (5), de même que les trajets de toutes sortes, présen- tent dès 1974 des caractéristiques similaires, mais moins affirmées, et les conservent au cours du dernier quart de siècle. Le travail professionnel et le sport, qui déjà en 1974 caractérisaient les plus diplômés, ont vu cette spécificité s'accentuer. En revanche, d'autres activités indiquent un changement des préférences induites par les clivages sociaux, notamment ceux qui sont en rapport direct avec le niveau de diplôme.

L'écoute de la radio ou de musique, ainsi que les pratiques religieuses, étaient en 1974 plutôt spécifiques des diplômés, en 1998 elles sont au contraire le fait des moins diplômés ; la faible

5. La pratique d'un instrument de musique a progressé surtout chez les jeunes. Mais elle décline fortement avec l'entrée sur le marché du travail (Donnat, 1999).

\section{Graphique III}

\section{Répartition des activités entre personnes très diplômés et peu diplômés, 1974-1998}

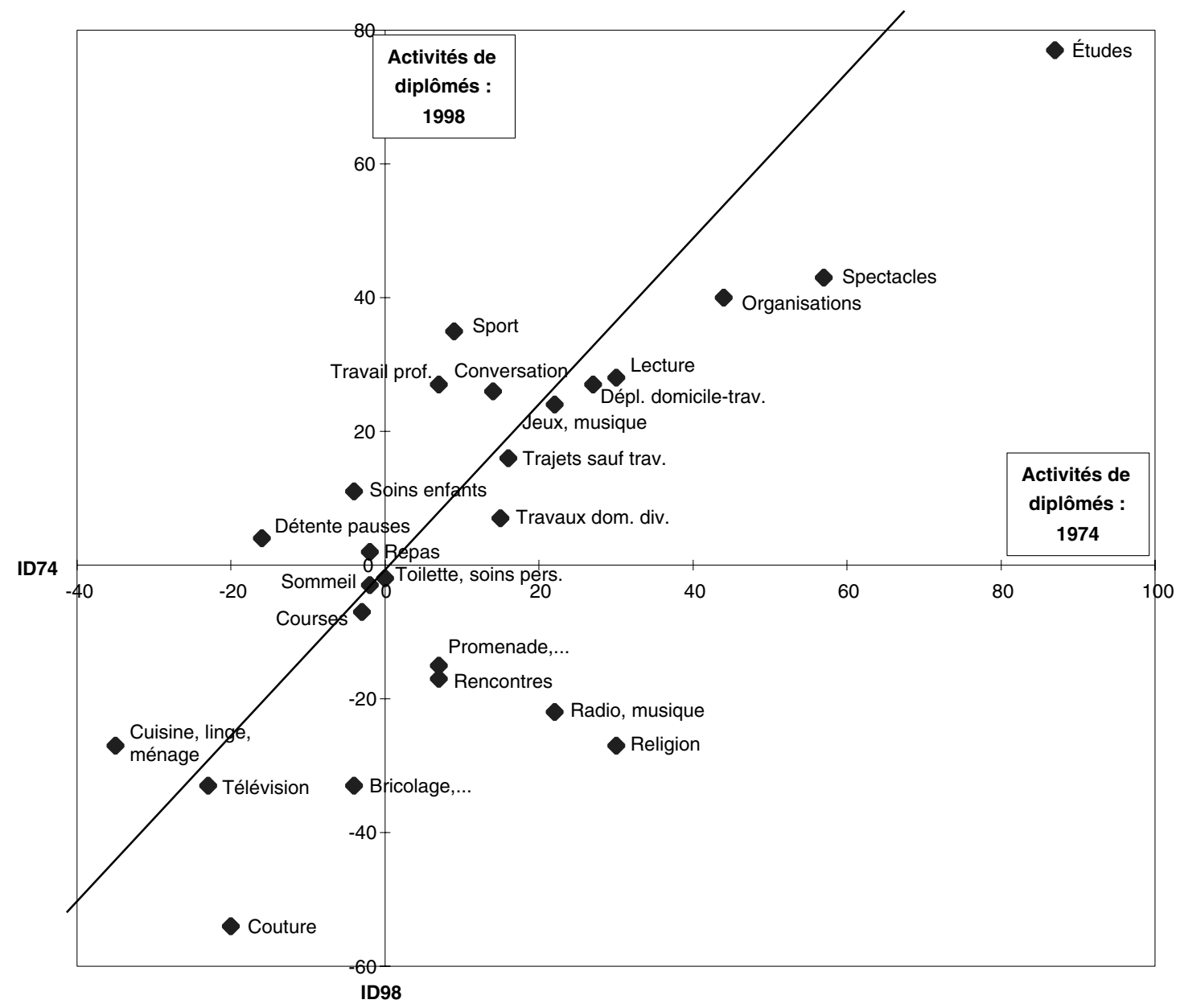

Lecture : on a porté en abscisses ID74, indice d'élasticité de la durée de l'activité par rapport au niveau de diplôme en 1974, et en ordonnées le même indice en 1998 (ID98). Pour la définition de cet indice, se reporter au tableau 4.

Champ : population urbaine de 18 à 64 ans.

Sources : enquêtes Emploi du temps, Insee. 
fréquence de la pratique religieuse ( $2 \%$ seulement des répondants décrivent de telles activités sur leur carnet) rend toutefois ces observations fragiles. Les rencontres, la promenade, plage, chasse et pêche se sont aussi affirmées comme des pratiques plutôt caractéristiques des milieux populaires.

Du moins les temps de conversation - activité peu différente des rencontres - et ceux de détente progressent-ils davantage parmi les diplômés que parmi les non diplômés. Ce groupe d'activité n'est socialement discriminant ni en 1974, ni en 1998.

\section{Les spectacles et les sorties dépendent plus du diplôme que du revenu}

Les spectacles et sorties constituent une activité très caractéristique des diplômés, généralement onéreuse. Cela amène à caractériser les effets propres du diplôme et du revenu en contrôlant les effets d'autres variables socio-démographiques - sexe, âge, statut d'emploi, situation de famille, type d'habitat - et du jour de la semaine (cf. tableau 9). En 1974, $3 \%$ des répondants mentionnent une telle activité sur leur carnet, en 1998, 4,5\%. Pour ceux qui s'y adonnent, la durée moyenne d'un spectacle est de 2 h 04 en 1974 et de 2 h 13 en 1998. Les effets sont calculés par des régressions logistiques rendant compte des variations des chances d'aller au spectacle plutôt que de ne pas y aller. En 1974 la probabilité de sortir est significativement plus élevée chez les hommes que chez les femmes, l'écart s'atténue en 1986 et n'est plus significatif en 1998 - la tendance à une convergence entre emplois du temps masculins et féminins s'observe donc ici encore au trois enquêtes. Les moins de 25 ans sortent significativement plus que les plus âgés : la jeunesse est, par excellence, l'âge d'une «culture de sorties » (Donnat, 1999). Un logement en immeuble collectif correspond lui aussi à une plus grande propension à aller au spectacle ou à sortir, par opposition à la maison individuelle, espace de repli sur des activités domestiques (cet écart est significatif en 1986 et en 1998). À chacune des dates,

Tableau 9

Sortir ou aller au spectacle : effets ajustés de huit variables explicatives

\begin{tabular}{|c|c|c|c|c|c|c|}
\hline & \multicolumn{2}{|c|}{1974} & \multicolumn{2}{|c|}{1986} & \multicolumn{2}{|c|}{1998} \\
\hline & Coefficient & Test & Coefficient & Test & Coefficient & Test \\
\hline Constante & $-4,96$ & $p<0,001$ & $-3,90$ & $p<0,001$ & $-4,12$ & $\mathrm{p}<0,001$ \\
\hline $\begin{array}{l}\text { Homme } \\
\text { Femme }\end{array}$ & $\begin{array}{l}0,53 \\
\text { Réf. }\end{array}$ & $p<0,001$ & $\begin{array}{l}0,29 \\
\text { Réf. }\end{array}$ & $p<0,001$ & Réf. & n.s. \\
\hline $\begin{array}{l}18 \text { à } 24 \text { ans } \\
25 \text { à } 34 \text { ans } \\
35 \text { à } 44 \text { ans } \\
45 \text { à } 54 \text { ans } \\
55 \text { à } 64 \text { ans }\end{array}$ & $\begin{array}{c}1,06 \\
0,66 \\
\text { Réf. } \\
- \\
-\end{array}$ & $\begin{array}{c}\mathrm{p}<0,001 \\
\mathrm{p}<0,001 \\
\text { n.s. } \\
\text { n.s. }\end{array}$ & $\begin{array}{c}1,07 \\
- \\
\text { Réf. } \\
- \\
-0,44\end{array}$ & $\begin{array}{c}\mathrm{p}<0,001 \\
\text { n.s. } \\
\text { n.s. } \\
\mathrm{p}<0,01\end{array}$ & $\begin{array}{c}0,85 \\
0,30 \\
\text { Réf. } \\
- \\
-\end{array}$ & $\begin{array}{c}p<0,001 \\
p<0,05 \\
\text { n.s. } \\
\text { n.s. }\end{array}$ \\
\hline $\begin{array}{l}\text { Occupé } \\
\text { Chômeur ou inactif }\end{array}$ & Réf. & n.s. & $\begin{array}{l}0,24 \\
\text { Réf. }\end{array}$ & $p<0,01$ & Réf. & n.s. \\
\hline $\begin{array}{l}\text { CEP, sans diplôme } \\
C A P, B E P, B E P C \\
\text { Bac et plus }\end{array}$ & $\begin{array}{c}- \\
\text { Réf. } \\
0,65\end{array}$ & $\begin{array}{c}\text { n.s. } \\
p<0,001\end{array}$ & $\begin{array}{l}-0,30 \\
\text { Réf. } \\
0,36\end{array}$ & $\begin{array}{l}p<0,001 \\
p<0,001\end{array}$ & $\begin{array}{c}-0,47 \\
\text { Réf. } \\
0,66\end{array}$ & $\begin{array}{l}\mathrm{p}<0,001 \\
\mathrm{p}<0,001\end{array}$ \\
\hline $\begin{array}{l}\text { Revenu, premier quartile } \\
\text { Revenu, } 2^{e} \text { et } 3^{e} \text { quartiles } \\
\text { Revenu, } 4^{\mathrm{e}} \text { quartile }\end{array}$ & Réf. & $\begin{array}{l}\text { n.s. } \\
\text { n.s. }\end{array}$ & $\begin{array}{l}-0,26 \\
\text { Réf. } \\
-\end{array}$ & $\begin{array}{l}\mathrm{p}<0,05 \\
\text { n.s. }\end{array}$ & $\begin{array}{l}-0,40 \\
\text { Réf. } \\
0,27\end{array}$ & $\begin{array}{l}p<0,001 \\
p<0,01\end{array}$ \\
\hline $\begin{array}{l}\text { Personne de référence } \\
\text { ou conjoint, avec enfant(s) } \\
\text { Autre sans enfant }\end{array}$ & Réf. & n.s. & $\begin{array}{l}-0,34 \\
\text { Réf. }\end{array}$ & $p<0,001$ & Réf. & n.s. \\
\hline $\begin{array}{l}\text { Maison individuelle } \\
\text { Immeuble collectif }\end{array}$ & Réf. & n.s. & $\begin{array}{l}-0,29 \\
\text { Réf. }\end{array}$ & $p<0,001$ & $\begin{array}{l}-0,22 \\
\text { Réf. }\end{array}$ & $\mathrm{p}<0,05$ \\
\hline $\begin{array}{l}\text { Lundi au vendredi } \\
\text { Samedi } \\
\text { Dimanche }\end{array}$ & $\begin{array}{l}\text { Réf. } \\
1,23 \\
1,95\end{array}$ & $\begin{array}{l}p<0,001 \\
p<0,001\end{array}$ & $\begin{array}{l}\text { Réf. } \\
1,22 \\
1,59\end{array}$ & $\begin{array}{l}p<0,001 \\
p<0,001\end{array}$ & $\begin{array}{l}\text { Réf. } \\
1,33 \\
1,55\end{array}$ & $\begin{array}{l}p<0,001 \\
p<0,001\end{array}$ \\
\hline
\end{tabular}

Lecture : régressions logistiques. Toutes choses égales par ailleurs (âge, statut d'emploi, diplôme, revenu, statut parental, habitat, jour de la semaine), en 1974 un homme a plus de chances qu'une femme de sortir ou d'aller au spectacle; en 1998, cet écart n'est plus significatif.

Champ : population urbaine âgée de 18 à 64 ans en 1974, 1986 et 1998.

Sources : enquêtes Emploi du temps, Insee. 
les sorties sont significativement plus fréquentes le samedi ou le dimanche qu'en semaine. Le statut d'emploi et la situation familiale n'ont d'effet propre significatif qu'en 1986.

Une fois contrôlés les effets du sexe, de l'âge, du statut d'emploi, du type de ménage, de l'habitat et du jour de la semaine, les effets nets du revenu sur la probabilité d'aller au spectacle ou de sortir sont plus faibles que ceux du diplôme. L'écart entre les bacheliers et les titulaires de diplômes de niveau intermédiaire est à peu près le même en 1974 et en 1998. En revanche, celui entre les non diplômés et le niveau intermédiaire, non significatif en 1974, le devient ensuite. Les effets du revenu n'étaient pas significatifs en 1974 , ils se manifestent de plus en plus nettement en 1986 puis en 1998. En 1998, la probabilité nette d'aller au spectacle est presque trois fois plus forte chez les bacheliers que chez les sans diplôme ou les titulaires du certificat d'études. Elle est presque deux fois plus forte chez les titulaires de revenus du quartile supérieur que chez ceux du quartile inférieur. Spectacles et sorties sont des loisirs qui, pour ceux qui sont plus à court de temps que d'argent, ont l'avantage de pouvoir combiner sur une durée relativement brève, du divertissement ou de la culture, du restaurant, de la sociabilité, sans avoir à supporter les préparatifs au domicile et les rangements après la fête.

\section{BIBLIOGRAPHIE}

Atkinson T., Glaude M. et Olier L. (2001), « Les inégalités économiques », in Inégalités économiques, La Documentation française (« Rapports du Conseil d'Analyse Économique », n³3).

Baudelot C. et Gollac M. (2001), « La perméable frontière du bonheur », Premiers résultats d'une enquête Insee-Dares, Libération, 17 septembre.

Chenu A. (2001), « Vieillissement, genre et inégalités sociales dans la France des années 19801990 : le prisme des emplois du temps », Cahiers du genre, $\mathrm{n}^{\circ} 31, \mathrm{pp} .9-37$.

Donnat O. (1999), Les amateurs, enquête sur les activités artistiques des Français, ministère de la Culture.

Dumazedier J. (1962), Vers une civilisation des loisirs?, Éditions du Seuil, Paris.

Dumazedier J. (1988), Révolution culturelle du temps libre, 1968-1988, Klincksieck, Paris.

Fermanian J.-D. (1999), « Le temps de travail des cadres », Insee première, $\mathrm{n}^{\circ} 671$.

Fougère D. et Kramarz F. (2001), «La mobilité salariale en France de 1967 à 1999 », in Inégalités économiques, La Documentation française, (« Rapports du Conseil d'Analyse Économique », n³3).

Godechot O., Lurol M. et Méda D. (1999), « Des actifs à la recherche d'un nouvel équilibre entre travail et hors travail », Premières Synthèses, Dares, $\mathrm{n}^{\circ} 20.1$
Gershuny J. (2000), Changing Times: Work and Leisure in Postindustrial Society, Oxford University Press.

Herpin N. et Verger D. (2000), La consommation des Français (deux tomes), Éditions La Découverte.

Insee (1990a), Enquête sur les emplois du temps (1974-1975). Dessins des fichiers - Dictionnaires des codes.

Insee (1990b), Enquête sur les emplois du temps (1985-1986). Fichier LEDA-A. Dessins des fichiers - Dictionnaires des codes (4 tomes).

Linder S. (1982), La ressource la plus rare, trad. fr., Les éditions Bonnel, Paris, (première édition New York, 1970).

Robinson J.P. et Godbey G. (1999), Time for Life: the Surprising Ways Americans Use their Time, University Park: Pennsylvania State Press (2e édition).

Schor J. (1993), The Overworked American: the Unexpected Decline of Leisure, NY Basic Books.

Singly F. de et Thélot C. (1988), Gens du privé, gens du public, la grande différence, Éditions Dunod.

Vallet L.-A. (1999), « Quarante années de mobilité sociale en France. L'évolution de la fluidité sociale à la lumière de modèles récents », Revue Française de Sociologie, 40-2.

Yonnet P. (1999), Travail, loisir : temps libre et lien social, Éditions Gallimard. 
